\title{
Retrieval of Spatial Join Pattern Instances from Sensor Networks*
}

\author{
Man Lung Yiu \\ Department of Computer Science \\ Aalborg University \\ DK-9220 Aalborg, Denmark \\ mly@cs.aau.dk
}

Nikos Mamoulis

Department of Computer Science

University of Hong Kong

Pokfulam Road, Hong Kong

nikos@cs.hku.hk

\author{
Spiridon Bakiras \\ Dept. of Math. and Comp. Science \\ John Jay College \\ City University of New York \\ sbakiras@jjay.cuny.edu
}

\begin{abstract}
We study the continuous evaluation of spatial join queries and extensions thereof, defined by interesting combinations of sensor readings (events) that co-occur in a spatial neighborhood. An example of such a pattern is "a high temperature reading in the vicinity of at least four highpressure readings". We devise protocols for 'in-network' evaluation of this class of queries, aiming at the minimization of power consumption. In addition, we develop cost models that suggest the appropriateness of each protocol, based on various factors, including selectivity of query elements, energy requirements for sensing, and network topology. Finally, we experimentally compare the effectiveness of the proposed solutions on an experimental platform that emulates real sensor networks.
\end{abstract}

${ }^{*}$ Work supported by grant HKU 7155/06E from Hong Kong RGC.

${ }^{\dagger}$ A preliminary version of this work appeared in [25], available at http://www.cs.aau.dk/ mly/ssdbm07_senpat.pdf 


\section{Introduction}

Advances in computer hardware have brought to availability small and relatively cheap devices forming a powerful network that interacts and collects information from the environment, where it is deployed [27]. Sensor networks have several applications, including environmental monitoring $[15,13]$, control/maintenance of industrial infrastructure [1], military applications [20], structural monitoring [17], etc. Recently, the problem of evaluating queries over a sensor network has attracted significant research interest from the database community, leading to the development of two research DBMS prototypes [24, 14]. These systems provide to the user an interface, via which queries are expressed in a declarative way; the user needs not deal with how queries are evaluated. Suitable extensions of SQL were proposed with clauses that consider the special features of sensor networks. These features include the transient, on-demand nature of sampled data, extended lifetime of continuous (non-transient) queries, sampling rate or compression of sensor readings, event-triggered queries, etc.

The main focus of existing work on sensor networks has been the minimization of power consumption at sensor nodes, during query evaluation. Sensors are usually battery-operated and they are often deployed in hostile environments or rough terrains, where the network runs unsupervised for long time intervals. Thus, power is of utmost importance, since it is directly related to the longevity of the network. Previously studied topics include the energy-efficient retrieval of aggregations or data summaries $[13,5,3,7,6,19]$, the derivation and maintenance of data models that describe the data distribution $[8,4]$, and the optimal in-network placement of operators or filter predicates on the sensed values $[14,2,1,22]$. To our knowledge, there is no prior work for in-network evaluation of queries that spatially correlate measurements from different sensors. An example of such a query (taken from [3]) is "generate a notification whenever two sensors within 5 yards from each other simultaneously measure an abnormal temperature". A spatial pattern query retrieves sets of sensors (pairs in this example), whose readings qualify some selection predicates (e.g., abnormal temperatures) and their locations qualify some pairwise distance predicates (e.g., within five yards). Data analysts may be interested in the on-line identification of pattern instances that occur 
rarely in the environments where sensors are deployed and may indicate exceptional events. For instance, an unusually high temperature detected in the vicinity of multiple low-humidity readings may indicate high chance of a fire break in the local area, where the pattern is detected. Another application of spatial pattern queries is the prediction of weather phenomena based on spatial combinations of sensor readings.

A straightforward way to evaluate spatial pattern queries is to program the sensors to transmit their readings together with their locations to a central basestation (via a routing tree [10, 14]), where their spatial associations are validated. Although this approach is easy to implement, it may waste more energy than necessary, as sensor readings that are not part of query results may be sent all the way up to the root. Motivated by the lack of effective evaluation protocols for spatial pattern queries, in this paper, we study this problem in depth, focusing on (i) filtering techniques for readings that do not participate in the result, (ii) in-network computation of query results. We propose optimized evaluation protocols for binary spatial joins and more complex query patterns and compare them for different problem parameters. Our solutions are orthogonal to snapshotbased schemes (e.g., [11]), which apply query evaluation only to a small (self-maintained) sample of the network and to techniques that summarize sensor readings over long time intervals before applying query evaluation on them (e.g., [6]). The contributions of this paper can be summarized as follows:

- We identify the interesting class of spatial pattern queries. We formally define them and discuss how they can be expressed using the language extensions of [14].

- We propose energy-efficient protocols for in-network evaluation of spatial pattern queries. In addition, we provide cost models which can be used by a query optimizer to determine a suitable evaluation method based on query parameters and data statistics.

- We experimentally evaluate the effectiveness of the proposed techniques by tuning various parameters, including query selectivity, network size, topology and density, sampling cost, etc. 
The remainder of the paper is organized as follows. Section 2 reviews related work. Section 3 formally defines spatial pattern queries. In Section 4, we describe in detail the proposed solutions, and analyze their costs in Section 5. Section 6 discusses the evaluation of variants and extensions of pattern queries, as well as advanced issues, like multiple query evaluation. Section 7 experimentally demonstrates the applicability and efficiency of our techniques. Finally, Section 8 concludes the paper.

\section{Background and Related Work}

The special characteristics of a sensor network compared to a generic wireless network are (i) the limited resources of nodes (energy, communication range, network bandwidth and capacity), (ii) unreliable communication with high packet loss rates and frequent node failures, and (iii) unsupervised nature with nodes placed at hostile environments (e.g., remote areas, war fields, etc.). Thus, query evaluation techniques for sensor networks aim at minimizing the energy cost, subject to the constraints of the network (e.g., communication range, maximum data volume that can be sent by a node at a cycle, etc.). Besides, sensor networks are inherently redundant (i.e., dense), in order to keep the network connected after node failures and increase the reliability of sensed information.

Query evaluation in sensor networks is performed in two steps [10, 24, 14]. Suppose that the query should collect the readings from all sensors. The query is registered at a basestation, which is connected to a root node $r$. In the first step, the query is disseminated to the sensors, and a spanning tree of the network, rooted at $r$ is dynamically constructed. If a node receives the query for the first time, it selects one of the senders as its parent in the tree and broadcasts the query. Otherwise, the message is ignored. The resulting communication (or routing) tree is used to acquire sensor readings related to the query, up to the basestation. Delivery of sensor readings (or query results) to the root is performed in multiple phases. During a specific phase, a level of the tree sends and the level above listens and receives information addressed for it. Finally, the root collects all readings and sends them to the basestation. 
Queries over sensor networks are usually continuous, i.e., they remain active for a lengthy time interval (e.g., minutes, hours). Otherwise, the cost for disseminating the query may not be compensated. Frequent instantaneous queries are best processed if the network operates in a push-based manner; sensors periodically and unconditionally collect measurements and route them to a basestation, where queries are registered and evaluated as queries over streaming data. For example, in the work of [9], efficient algorithms are developed for processing continuous constraint queries at a centralized basestation, without considering communication cost in the underlying infrastructure (e.g., sensor network). In this paper, we exploit in-network evaluation techniques in order to minimize power consumption of the sensor network for processing continuous queries. Next, we review work on (continuous) query evaluation on sensor networks.

\subsection{Aggregation and summarization}

Madden et al. [13] proposed a simple, but powerful protocol for computing common aggregate functions (e.g., count, sum, max, min). Each sensor combines the information received by its children with its own measurement to derive and send data of constant size, capturing a partial computation of the aggregate function. In [5], a multi-path algorithm for computing aggregates is presented to reduce communication errors as multiple parents may hear and aggregate the information broadcast by a single child. [16] proposes a hybrid method that combines the tree topology of [13] with the ring network topology of [5]. Besides, [7] describes a method for pushing error tolerance in network nodes, in order to avoid sending information if the aggregate is within some error bound. The problem of redistributing the error tolerance among nodes in order to minimize the overall error at dynamic environments is also studied. A similar approach was independently proposed in [19]. To minimize network communication, [6] presents a methodology for in-network compression of multiple (time-series) signals generated by sensors (e.g., one for temperature, one for humidity, etc.). The rationale is that measurements observed at the same node are likely to follow similar trends. Soheili et al. [21] focused on the processing of spatial aggregation query, which derives the aggregate (e.g., average) of sensor values (e.g., temperatures) in a user-defined spatial window $W$. They developed a distributed and hierarchical structure on the sensor network 
such that each node maintains the enclosing rectangle of its descendants in the routing tree. Energy consumption reduction is achieved by keeping irrelevant nodes (for query processing of $W$ ) asleep.

\subsection{Data models, snapshots, and filters}

An alternative to continuously collecting and processing sensor data (which drains the network energy resources), is to define and maintain simple data models (e.g., mixtures of Gaussians) for the data distribution $[8,4]$. These models, potentially combined with exact readings, provide query answers with some approximation confidence. Besides, [11] describes a framework for dynamically selecting and maintaining representatives in a redundant sensor network. The set of representatives (snapshot) plays the role of a dynamic sample that can answer queries cheaply and approximately.

Another class of problems is the distribution of filters or database operators in the routing tree of a sensor network. [22] studies the optimal placement of query operators (e.g., selection predicates), in order to minimize (i) the communication cost for information that does not end up in the query result and (ii) the computational burden at lower tree levels (assuming that lower-level nodes have reduced computational capabilities). [2] focuses on the assignment of operators that correlate measurements from two (apriori defined) spatial regions. [26] examines a similar problem and applies synopses of sensor values to eliminate unqualified readings that cannot lead to results. Assuming that tuples originated from sensors in two different spatial regions, [18] develops solutions for routing and joining those tuples in the sensor network. On the other hand, our problem searches for rare spatial associations of (instantaneous) events, anywhere in the network map.

In another direction, [1] studies continuous joining a table of predicates (e.g., 'humidity $>50^{\circ} \mathrm{C}$ ') with the sensed values. If the table is small enough to be stored at each node, it acts a filter that prevents non-qualifying readings to be sent to the basestation. If the table cannot fit in a node's local memory, it is placed at neighboring nodes and the predicates are evaluated in a distributed fashion. Yang et al. [23] examine continuous self-join processing on the tuples generated from the sensor network; two tuples are joined together if they satisfy the join predicate and stay in the same 
time window. In contrast to $[1,23]$, the queries we study do not simply consider sensor values; they also have to satisfy a spatial pattern, which will be defined formally in the next section.

The closest work to ours is [12], which reports pairs of sensor events located within a given distance range, and reduces communication cost by a distributed routing index. The sensors record past events in their neighborhood which help to predict future occurrences of them at other locations of the map. Messages are then routed based on these predictions. As the author suggests, the index is appropriate for applications where events correspond to moving objects with well-estimated future locations. Our focus, on the other hand is on arbitrary, instantaneous, ad-hoc events. In addition, the methodology of [12] relies heavily on the regular grid networks and may not be applicable to arbitrary network topologies.

\section{Problem Formulation}

Let $\mathcal{S N}$ be a network of $N$ sensors. Each sensor $s \in \mathcal{S N}$ is associated with a spatial location ${ }^{1}$ s.loc, and can produce a set s.m of measurements (e.g., temperature, humidity, etc.) for the spatial region around it (different sensors might produce different sets of readings, in general).

We adopt the framework described in Section 2, where users register continuous queries at a basestation and a routing tree is created to acquire results (or readings that are processed at the base). Each registered query is associated with: (i) a lifetime (e.g., 2 hours), during which it is active and continuously produces results from the sampled measurements, and (ii) an epoch duration (e.g., 10 seconds), every which the network samples measurements. In other words, queries apply to instances of the network at different timestamps (for every epoch).

A binary spatial pattern query identifies pairs of sensors, for which (i) the readings qualify some particular selection predicates and (ii) the locations are no further than a particular distance from each other. An example of such a query is "find pairs of sensors $\left\langle s_{1}, s_{2}\right\rangle$, such that $s_{1}$.temperature $>50^{\circ} \mathrm{C}$, $s_{2}$.humidity $<40 \%$, and distance $\left(s_{1}, s_{2}\right)<10 \mathrm{~m}$ ". A generalized spatial pattern query (formally de-

\footnotetext{
${ }^{1}$ We assume that the locations of sensors are known to them. They could be constant and apriori defined (for stationary, manually placed sensors), or detected by GPS devices placed on the sensors.
} 


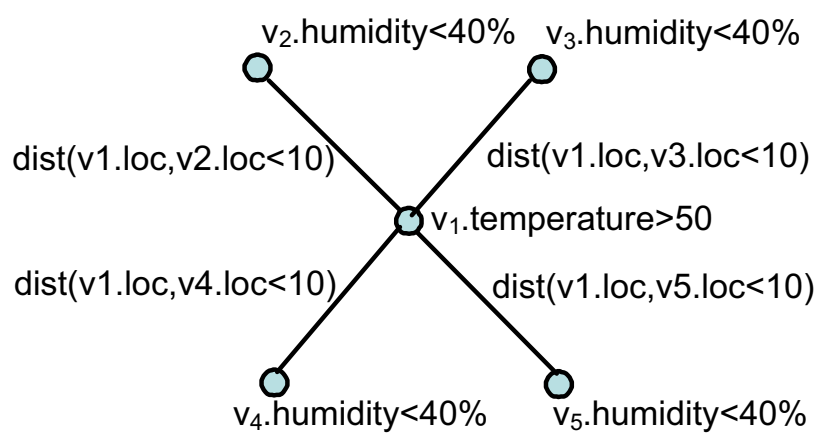

Figure 1: A spatial pattern query

fined below) returns sets of sensors, whose values and locations qualify some selection predicates and binary distance predicates, respectively.

Definition 1 A spatial pattern query $Q$ consists of a set Q.V of variables, a set Q.P of selection predicates, and a set $Q . \mathcal{B}$ of binary constraints. Each variable $v_{i} \in \mathcal{V}$ is associated to a selection predicate $P_{i}$. A pair $\left\langle v_{i}, v_{j}\right\rangle$ of variables $v_{i}, v_{j} \in \mathcal{V}, i \neq j$ may be associated with a binary spatial predicate $B_{i j}$. A result of $Q$ is set of assignments $\left\{\forall v_{i} \in \mathcal{V}: v_{i} \leftarrow s_{i}, s_{i} \in \mathcal{S N}\right\}$, such that (i) for all $v_{i}, P_{i}\left(v_{i} . m\right)$ is satisfied and (ii) for all variable pairs $\left(v_{i}, v_{j}\right)$ with a binary predicate, $B_{i j}\left(v_{i} . l o c, v_{j} . l o c\right)$ is satisfied.

Figure 1 shows an example of a spatial pattern query $q$ modeled by a graph. Each node in the graph corresponds to a variable, whose values are constrained by a selection predicate. Edges correspond to binary spatial predicates (i.e., distance constraints). In natural language, $q$ could be expressed as "a high-temperature reading $\left(>50^{\circ} \mathrm{C}\right)$ in the vicinity of four low-humidity readings $(<40 \%)$ ". A local group of sensors whose readings satisfy this query could indicate an area that requires special attention (e.g., high chances of fire, if a forest).

Spatial pattern queries can be easily expressed in the extended SQL of [14], assuming that the language supports spatial functions (i.e., distance). The query variables (defined in the FROM clause) are instantiated by tuples of the Sensors table and the selection while join predicates (i.e., distance constraints) are connected by AND in the WHERE clause. Although Definition 1 is generic enough to define queries of arbitrary graphs and constraints, we confine our attention mainly to binary joins and to extended patterns that form "star" graphs (like the one in Figure 1), where 
a centric feature (e.g., high temperature) is correlated to a number of other features (e.g., low humidity) in its surrounding environment. Such patterns were shown important in spatial analysis applications and are more intuitive than queries that combine variables in an arbitrary graph. The centric feature models a point of interest (e.g., high fire risk area, expensive equipment) which should trigger an alert whenever its local measurements and the conditions in the region around it form an abnormal combination.

\section{Proposed Methods}

In this section, we explore the applicability of several methods for computing spatial pattern queries in a sensor network. We divide the evaluating protocols in two classes. The class of acquisitional protocols collect sensor measurements via the communication tree and apply query evaluation at the basestation. Filters are placed at nodes that generate or relay data to minimize the transferred volume. The second class of distributed protocols apply in-network query evaluation and send the results to the basestation (again using the tree). We start by discussing the simple case of a binary spatial join with distance constraint smaller than the communication range of the nodes. Then, we extend the suggested protocols for more complex queries and multi-hop distance constraints.

\subsection{Single-hop binary joins}

We first focus on binary join patterns that are sensor pairs $\left\langle s_{i}, s_{j}\right\rangle$, such that $P_{1}\left(s_{i} . m\right), P_{2}\left(s_{j} . m\right)$ are satisfied, and distance $\left(s_{i} . l o c, s_{j} . l o c\right) \leq c$, where $c$ is smaller than the radio communication range $^{2}$ between two nodes. For the ease of exposition, we denote a binary join query in our context by the triplet $\left\langle P_{1}, P_{2}, c\right\rangle$.

\subsubsection{Brute-force acquisitional protocol}

The straightforward way to evaluate the query is to program all sensors to sense the measurements relative to selection predicates $P_{1}$ and $P_{2}$, at every epoch, and send this information to the bases-

\footnotetext{
${ }^{2}$ Without loss of generality, we assume that all sensors have the same communication range. Our protocols and filtering techniques can be easily adjusted for the generic case.
} 
tation, which evaluates the spatial join locally. A simple optimization that reduces the number of unnecessary values transmitted to the base is to "push-down" the selection predicates at the nodes (as suggested in $[14,1]$ ). In our example, temperature and humidity are sensed by all sensors but only high temperature and low humidity values (i.e., those that qualify the selection predicates) are sent to the base. In order to minimize the transferred data, we only transmit the location of a qualifying node (or its identifier, if nodes have fixed locations) and two bits that indicate which predicate(s) the node qualifies (e.g., 10 implies that $P_{1}$ is qualified, but $P_{2}$ is not). Sensors are synchronized such that only two consecutive levels of the tree are active at the same phase (while the remaining nodes are sleeping), as discussed in Section 2. When a lower-level node senses and transmits data (if not filtered by $P_{1}$ or $P_{2}$ ) to its parent, its parent listens, reads and combines its readings with those of its children; the combined readings are then sent to the upper level during the next phase. We denote this simple, but generic protocol by AQB (i.e., the first 'acquisitional' protocol).

As an example, consider the sensor network depicted in Figure 2. Nodes within communication range from each other are connected by edges. Solid edges denote the structure of the communication tree (rooted at node $s_{3}$ ). The values next to the nodes denote the (current) local temperature $(\mathrm{T})$ and humidity $(\mathrm{H})$ conditions. Let $P_{1}={ }^{\circ} \mathrm{T}>50$ ', $P_{2}={ }^{\prime} \mathrm{H}<40$ ', and $c$ equals the sensor communication range (one hop). Nodes $s_{1}$ and $s_{2}$ qualify $P_{1}$, whereas $s_{6}$ and $s_{7}$ qualify $P_{2}$. The only join result is $\left\langle s_{2}, s_{6}\right\rangle$. In the first phase of the cycle, $s_{1}, s_{4}$, and $s_{7}$ (level-3 nodes) sense their values, apply the predicates and $s_{1}$ sends $\left(s_{1} . l o c, 10\right)$ (i.e., only $P_{1}$ is satisfied) to its parent (i.e., $\left.s_{5}\right)$. Similarly, $\left(s_{7} . l o c, 01\right)$ is sent to $s_{6}$. In the second phase, $\left(s_{2} . l o c, 10\right),\left(s_{1} . l o c, 10\right)$, and $\left\{\left(s_{7} \cdot l o c, 01\right),\left(s_{6} \cdot l o c, 01\right)\right\}$ are sent to the root, by $s_{2}, s_{5}$, and $s_{6}$, respectively. Finally, $s_{3}$ forwards all these tuples to the base, where the join result is computed.

\subsubsection{Pruner-based acquisitional protocol}

Protocol AQB may send more information than necessary to the base, as many tuples (e.g., ( $\left.s_{1} . l o c, 10\right)$ in Figure 2) are likely not to participate in the spatial join. In this section, we propose AQP, a protocol that improves upon AQB, by adding more sophisticated filters in the intermediate nodes of 


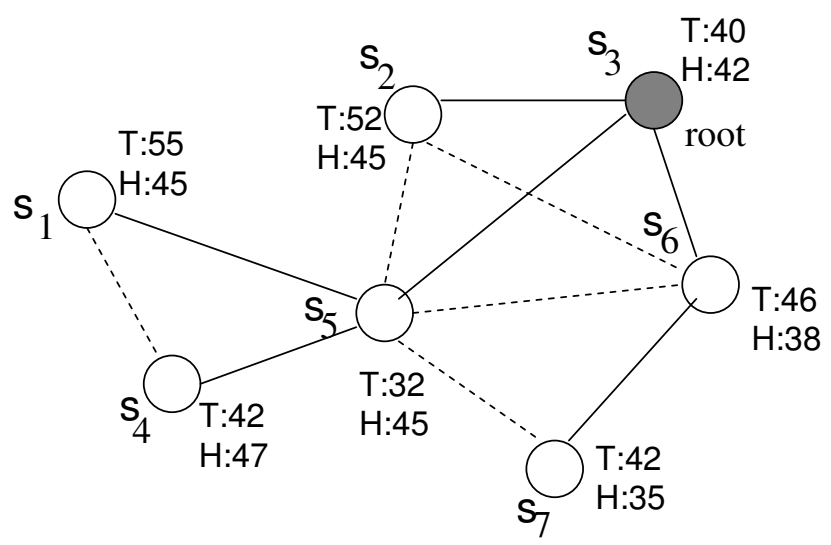

Figure 2: Join evaluation example

the tree. AQP is based on the observation that a sensed value (e.g., $m$ ) of a node $s_{i}$ which satisfies a selection predicate (e.g., $\left.P_{1}\right)$ can be pruned by an ancestor $a\left(s_{i}\right)$ of $s_{i}$, if (i) $a\left(s_{i}\right)$ collects all information about the spatial neighborhood of $s_{i}$ and (ii) no matching tuple (e.g., one that qualifies $P_{2}$ ) for the measurement has been collected by $a\left(s_{i}\right)$. For example, $\left(s_{1} . l o c, 10\right)$ in Figure 2 can be pruned by $s_{5}$, since any measurements that qualify $P_{2}$ within one hop from $s_{1}$ should have been collected or generated by $s_{5}$.

Formally, let $\left\langle P_{1}, P_{2}, c\right\rangle$ be a join query registered over a sensor network $\mathcal{S N}$. For each sensor $s \in \mathcal{S N}$, we define its neighborhood sensor set $L(s)$ as $L(s)=\left\{s^{\prime} \in \mathcal{S N} \mid \operatorname{dist}\left(s, s^{\prime}\right) \leq c\right\}$, and its descendant sensor set $B(s)$ as the set of sensors in its subtree (of the routing tree). The pruning technique applied in AQP is based on the Lemma 1 (with trivial proof):

Lemma 1 Let $s_{i}$ be a sensor satisfying $P_{1}\left(s_{i} . m\right)$. Let a $\left(s_{i}\right)$ be an ancestor of $s_{i}$, such that $L\left(s_{i}\right) \subseteq$ $B\left(a\left(s_{i}\right)\right)$. If there is no $s_{j} \in B\left(a\left(s_{i}\right)\right)$ satisfying $P_{2}\left(s_{j} . m\right)$, then $s_{i} . m$ cannot participate in an output tuple of $\left\langle P_{1}, P_{2}, c\right\rangle$. A symmetric argument holds for the measurements which qualify $P_{2}$.

For any sensor $s$, there is at least one ancestor (the root) for which $L(s) \subseteq B(a(s))$, thus we can apply this idea to prune measurements acquired from the network that do not participate in query results. The goal is to find the closest ancestor of $s$ to apply the filter, since, in this way, filtering effectiveness is maximized. For each node $s$, the pruner of $s$ (with respect to a query) is the nearest ancestor of $s$, whose subtree contains $L(s)$. In Figure $2, s_{5}$ is the pruner of $s_{1}$ and $s_{4}$. 
We design the following technique for determining pruners efficiently. It is applied only once while the query is disseminated and the routing tree is constructed. When a sensor node $s$ broadcasts the query, at the same time it collects ids/locations of its neighbors and determines $|L(s)|$ (the cardinality of $L(s)$ ), which is then broadcasted to all nodes in $L(s)$. Starting from the leaf nodes, each sensor $s$ sends up the communication tree a table consisting of $\left\langle s_{i},\left|L\left(s_{i}\right)\right|, 1\right\rangle$ tuples for all $s_{i} \in L(s)$ plus a $\langle s,|L(s)|, 1\rangle$ tuple for itself. Intermediate tree nodes merge the tables they receive from their children by summing their counters (the last field of the tuples). The first node which, after the aggregation, has a $\langle s,|L(s)|,|L(s)|\rangle$ tuple becomes the pruner for $s$ and does not forward the tuple to its parent node.

After this process, each node $s$ keeps a list of its prunees (i.e., nodes for which $s$ is the pruner). The difference between our improved acquisitional protocol AQP and the baseline AQB is that, in AQP, whenever $s$ retrieves information from its subtree regarding the join query, for each prunee that transmits a tuple $\tau$ qualifying $P_{1}\left(P_{2}\right), s$ checks whether there is a matching tuple for $P_{2}\left(P_{1}\right)$ in its acquired table, which also qualifies the distance predicate with $\tau$. If there is no such tuple, then $\tau$ is pruned from the data sent to the parent of $s$. AQP manages to filter early some node readings (e.g., $\left.\left(s_{1} . l o c, 10\right)\right)$ that do not qualify the join predicate.

\subsubsection{Distributed evaluation}

The class of distributed protocols aim at computing query results locally around network nodes and sending them to the basestation. Such a technique is expected to pay-off for low-selectivity ${ }^{3}$ joins, where many measurements that satisfy predicates $P_{1}$ or $P_{2}$ do not qualify the join condition. During the first stage of distributed evaluation, nodes that qualify $P_{1}$ and $P_{2}$ communicate and determine the join results. During the second stage, the routing tree is used to send the join results to the base.

Initially, all nodes sense the measurements related to $P_{1}$ and $P_{2}$. If a node $s_{i}$ qualifies $P_{1}$, it broadcasts its location to its neighborhood. If a node $s_{j}$ qualifies $P_{2}$, it listens for potential messages

\footnotetext{
${ }^{3}$ Low-selectivity joins output few results while high-selectivity joins produce many results.
} 
from nodes that qualify $P_{1}$. For each received message, $s_{j}$ produces a join result. Nodes that qualify neither $P_{1}$ nor $P_{2}$ remain asleep until the first stage terminates (they may have to wake and forward join results, during the second stage). Note that the roles of $P_{1}$ and $P_{2}$ could be interchanged; we denote by DS1 (DS2) the distributed protocol, where nodes qualifying $P_{1}\left(P_{2}\right)$ send messages and those qualifying $P_{2}\left(P_{1}\right)$ receive them and compute join results. Intuitively, DS1 should be preferred to DS2 when nodes that qualify $P_{1}$ are fewer than those qualifying $P_{2}$, since transmission is more expensive than listening and receiving [15].

As an example, consider again the network of Figure 2. In the first stage of the distributed protocol, measurements are collected, and (i) nodes $s_{1}$ and $s_{2}$ (qualifying $P_{1}$ ) broadcast their locations, (ii) nodes $s_{6}$ and $s_{7}$ (qualifying $P_{2}$ ) listen for potential messages, (iii) nodes $s_{3}, s_{4}$, and $s_{5}$ stay asleep. After node $s_{6}$ reads the transmission of $s_{2}$, the join result $\left\langle s_{2}, s_{6}\right\rangle$ is formulated. This is the only tuple that will be forwarded to the root at the second stage (result acquisition).

So far we have ignored the cost for sensing measurements at nodes, which is usually small compared to communication costs. For some measurements, however, this cost may be significant [14]. For cases where sensing for $P_{2}$ is significantly expensive, it might be beneficial to defer sensing and instruct all nodes to listen for $P_{1}$ messages. Only if a listener receives a message from a $P_{1}$ node, it performs expensive sensing for $P_{2}$ measurements. We denote this protocol by DS1'.

\subsection{Complex join queries}

We now consider more complex pattern queries, as described in Section 3. Queries correspond to star graphs, where the center sensor node should satisfy selection predicate $P_{C}$ and there are $k$ border nodes that should qualify $P_{B}$ within distance $c$ from the center. A star pattern query is simply denoted by a quadruple $\left\langle P_{C}, P_{B}, c, k\right\rangle$. As in Section 4.1, we assume that $c$ is at most equal to the radio range of the sensors.

Acquisitional protocols We can directly apply the brute-force acquisitional protocol AQB. Sensor readings that qualify $P_{C}$ or $P_{B}$ are unconditionally sent to the basestation, where the pattern is evaluated. In addition, we can adapt protocol AQP as follows. A tuple qualifying $P_{C}$ which has 
been generated by a node $s_{i}$ is filtered at node $\operatorname{pr}\left(s_{i}\right)$ (pruner of $s_{i}$ ) if there are less than $k$ tuples that qualify $P_{B}$ and reach $\operatorname{pr}\left(s_{i}\right)$ (otherwise, we know that there may be a query result that contains the tuple). A tuple qualifying $P_{B}$, which has been generated by a node $s_{i}$ is filtered at $\operatorname{pr}\left(s_{i}\right)$ if there is no tuple satisfying $P_{C}$ that reaches $\operatorname{pr}\left(s_{i}\right)$ (i.e., similar to binary join queries).

Distributed protocols A simple way to extend the distributed protocols for complex queries is to ask 'border' nodes (those qualifying $P_{B}$ ) broadcast their locations. At the same time 'centric' nodes (those qualifying $P_{C}$ ) listen for potential messages. If a centric node $s_{i}$, receives at least $k$ messages, we know that there is a query result centered at $s_{i}{ }^{4}$ The query result is sent to the base station through the routing tree, at the second stage of the protocol. We denote this protocol by DSB. An alternative protocol aims at minimizing the messages broadcast from border nodes; presumably more sensors qualify $P_{B}$ than $P_{C}$ for the pattern query to have small selectivity and correspond to an interesting, exceptional event. Protocol DSC asks nodes that qualify $P_{C}$ (center nodes) to send a message and nodes that qualify $P_{B}$ (border nodes) to listen. If a border node receives a message it sends a response with its location to its neighbors. Finally, center nodes listen for messages and those that hear from at least $k$ nodes send the query result to the base.

\subsection{Multi-hop queries}

Distance constraints longer than the radio range $h$ impose difficulties for distributed evaluation protocols. Given a node $s$, there is no bound for the number of hops required to find the nodes within distance $c(>h)$ from $s$. Nonetheless, for a relatively dense and uniform network, we could set an approximate upper bound $\lambda$ for this number. Let coverage $(c, \lambda)$ be the probability that two sensor nodes within distance $c$ are reachable within $\lambda$ hops. Figure 3 plots the coverage as a function of $\lambda$ on a typical random network (with the default parameter values discussed in Section 7). For instance, for the curve of " $c=3 h$ ", $c$ is set to 3 times of the radio range $h$. Observe that the coverage increases rapidly when $\lambda$ increases. In order to balance the coverage and energy consumption, we suggest to set $\lambda=\left\lceil\frac{c \sqrt{2}}{h}\right\rceil$ for multi-hop communication. We now discuss in more

\footnotetext{
${ }^{4}$ In fact, if $s_{i}$ receives $m>k$ messages, we have multiple query results, one for each $\left(\begin{array}{c}m \\ k\end{array}\right)$ combination of border nodes. Nonetheless all these results can be compressed to a single tuple containing $s_{i}$ and all qualifying border nodes.
} 
detail the protocols that can be applied for queries that involve multi-hop distances.

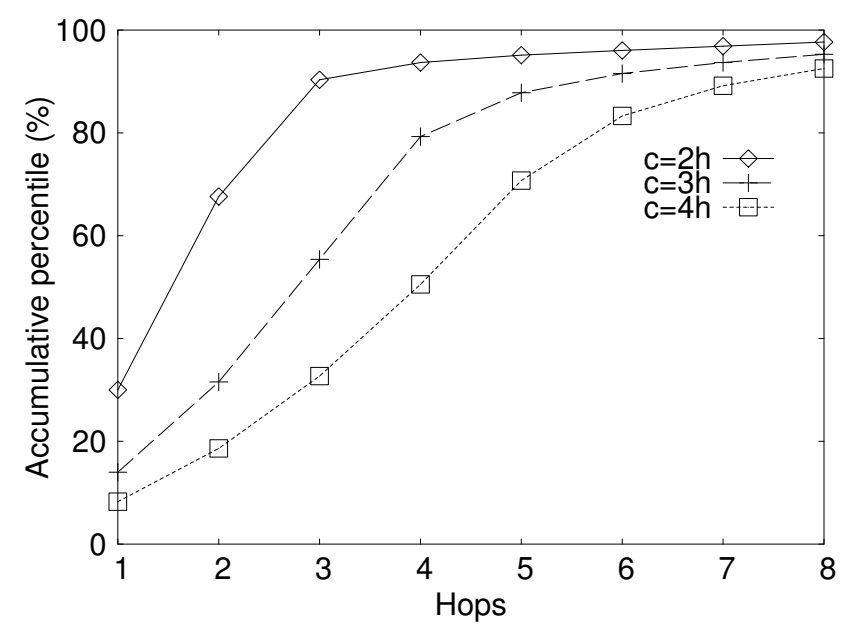

Figure 3: Coverage in multi-hop communication

Acquisitional protocols Since AQB does not apply any filtering or in-network evaluation, there is no difference than the method described in Section 4.1 for multi-hop queries. For AQP, the only difference is in the initialization of the query, at the stage when pruners are defined. Each node needs to determine the number of its $\lambda$-hop neighbors before sending it up the communication tree. This process requires flooding a large number of messages and it is more expensive than the simple 1-hop communication. However, it is performed only once, during the initialization of the routing tree and it is expected to pay off if the query has long lifetime.

Distributed protocols The distributed protocols described so far can be easily adapted for multihop queries, at the expense of higher communication cost, since the whole network may need to stay up in order to listen and relay potential messages, during the first stage (computation of query results). If $\lambda$ is large, the cost of flooding may be too high for distributed evaluation to pay-off. In such cases, the acquisitional protocols are expected to dominate.

A bi-directional distributed protocol For queries that are simple binary joins, we can apply a bi-directional distributed protocol (BD) in order to reduce message flooding during the first (computation) stage. Instead of asking nodes that qualify $P_{1}$ to flood their locations up to $\lambda$ hops (which are then received by listeners that qualify $P_{2}$ and converted to query results), we ask all 
nodes that qualify either $P_{1}$ or $P_{2}$ to send their locations and a pair of bits indicating the qualified predicates (i.e., the information sent by nodes to the base according to AQB/AQP). However, the flooding range is now reduced. Nodes that qualify $P_{1}$ send their messages up to $x$ hops $(x<\lambda)$ and nodes that qualify $P_{2}$ up to $\lambda-x$ hops. ${ }^{5}$ During this process all nodes of the network are up in order to listen and relay messages. If a node receives a message from both a $P_{1}$ node and a $P_{2}$ node, it formulates and caches the join result. In the second stage of the algorithm, nodes send the computed results up the tree to the basestation. Note that duplicate results could be computed, since the same pair of messages may be received by the same node. For instance, consider a query that seeks for high-temperature/low-humidity readings within $\lambda=2$ hops in the network of Figure 2. When BD is applied, all nodes that sense either high temperature $(>50)$ or low humidity $(<40)$ transmit their readings up to 1 hop (i.e., $x=1, \lambda-x=1)$. Both $s_{5}$ and $s_{6}$ then identify $\left.\left\langle s_{2}, s_{7}\right\rangle\right)$ as a result. Duplicate results are eliminated by merging operations at the second stage of the protocol, when all results are sent up the communication tree.

An interesting problem is to pick a value of $x$ such that the communication cost is minimized. In general, $x$ can take $\lambda+1$ values (the extreme cases $x=0, x=\lambda$ correspond to the uni-directional distributed protocols DS1 and DS2). Intuitively, $x$ should be chosen to minimize the expected expansion area $\pi\left(\operatorname{Sel}\left(P_{1}\right) \cdot x^{2}+\operatorname{Sel}\left(P_{2}\right) \cdot(\lambda-x)^{2}\right)$, where $\operatorname{Sel}\left(P_{i}\right)$ corresponds to the probability that a node qualifies $P_{i}$ (for $i=\{1,2\}$ ). In other words, if $P_{1}$ has low selectivity (few nodes qualify it) compared to $P_{2}$, nodes that qualify it should transmit far and nodes that qualify $P_{2}$ should transmit close in order to minimize network traffic.

\section{Cost Analysis}

In this section, we analyze the costs of the proposed protocols. We assume that the basestation maintains statistics about the sensed measurements. These statistics can be used to estimate the selectivity of selection and/or join predicates. They could either be collected by sampling readings from the whole network regularly, or by asking the sensors to compute and maintain local sum-

\footnotetext{
${ }^{5} \mathrm{~A}$ node that qualifies both predicates sends its message up to $\max \{x, \lambda-x\}$ hops.
} 


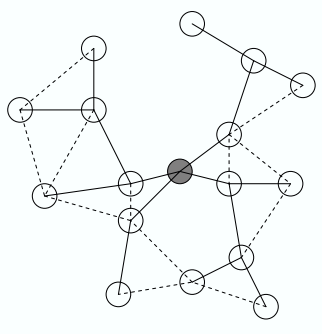

random

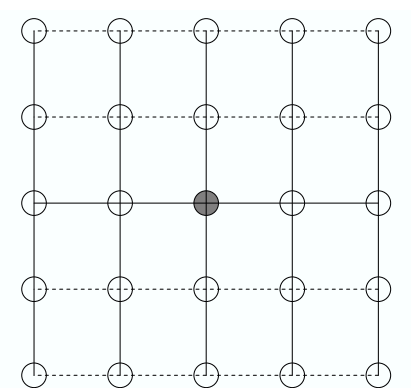

grid

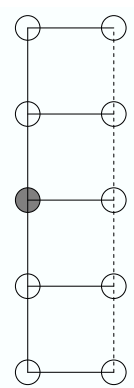

ladder

Figure 4: Three network topologies

maries, which are consolidated and sent to the base periodically (as in [6]). To provide examples throughout the analysis, we consider three network topologies, which simulate cases of randomly or manually placed sensors.

Figure 4 shows graphically the RANDOM, GRID, and LADDER networks. RANDOM represents the most common case with randomly deployed sensors, GRID network models the situation where sensors are distributed regularly, and LADDER corresponds to the scenario where sensors are placed along a road/track. In each network, the lines connect node pairs within 1-hop distance, assuming that the radio range equals the distance between two consecutive nodes in a row of the grid. The solid lines show potential routing trees, assuming that the root nodes are central to each network. The GRID and LADDER topologies are also shown in Figure 4. Due to space constraints, we confine our discussion to single-hop binary join queries.

Protocol AQB We start by analyzing the cost of protocol AQB, which is the simplest. Recall that each node $s$ generates a tuple if it qualifies either of $P_{1}$ and $P_{2}$. Let $\operatorname{Sel}\left(P_{1}\right)$ and $\operatorname{Sel}\left(P_{2}\right)$ be the selectivities of the two predicates (i.e., the probabilities to be satisfied). Let $E$ be the probability that a node generates a message. Assuming that the two predicates are independent, $E=1-\left(1-\operatorname{Sel}\left(P_{1}\right)\right)\left(1-\operatorname{Sel}\left(P_{2}\right)\right)$. Every node forwards tuples from itself and all its descendants to its parent in the routing tree. Let $|B(s)|$ be the number of nodes in the subtree $B(s)$ rooted at node $s$. Then, $s$ has $1-(1-E)^{|B(s)|}$ probability to transmit and when it does, it sends $|B(s)| \cdot E$ messages to its parent. Let $C$ be the maximum number of tuples that can fit in a packet. The total 
number of transmitted packets is expected to be:

$$
T_{\mathrm{AQB}}=\sum_{s \in \mathcal{S N}}\left(1-(1-E)^{|B(s)|}\right)\left\lceil\frac{|B(s)| \cdot E}{C}\right\rceil
$$

The number of received packets by sensor nodes is $R_{\mathrm{AQB}}=T_{\mathrm{AQB}}-\left\lceil\frac{N \cdot E}{C}\right\rceil$, since the root's packets are received by the base. After adding the costs for sampling two measurements per node, we derive the following formula:

$$
\operatorname{Cost}(\mathrm{AQB})=C_{T} T_{\mathrm{AQB}}+C_{R} R_{\mathrm{AQB}}+N\left(C_{S 1}+C_{S 2}\right)
$$

In Eq. 2, $C_{T}\left(C_{R}\right)$ and $C_{S 1}\left(C_{S 2}\right)$ are the costs for transmitting (receiving) a packet and sensing for $P_{1}\left(P_{2}\right)$. Note that we ignore the processing cost, which is insignificant (only few calculations are performed at each node). We assume that $|B(s)|$, for each $s$ is known by the optimizer. For random networks, this information can be forwarded to the base after query dissemination.

Protocol AQP Protocol AQP is similar to AQB, except that tuples may be filtered out at pruner nodes. Let $E_{1}$ be the probability that a node satisfies $P_{1}$ and no node within distance $c$ from it satisfies $P_{2}$. We can compute $E_{1}$ if we know the average size of a neighborhood. Let $c$ be the join distance. In a random network, the expected number of nodes within distance $c$ from a random node (excluding itself) is given by $\rho=N \cdot \pi c^{2} / A$, where $N$ is the number of nodes, and $A$ is the area of the workspace. Then, $E_{1}=\operatorname{Sel}\left(P_{1}\right) \cdot\left(1-\operatorname{Sel}\left(P_{2}\right)\right)^{\rho+1}$. Similarly, we can derive $E_{2}=\operatorname{Sel}\left(P_{2}\right) \cdot\left(1-\operatorname{Sel}\left(P_{1}\right)\right)^{\rho+1}$. The probability that a node satisfying either $P_{1}$ or $P_{2}$ does not participate in a join result is $E_{1}+E_{2}$, since the two events are mutually exclusive (a node is within distance $c$ from itself). Thus, the probability that a generated tuple will be pruned is $\frac{E_{1}+E_{2}}{E}$ and this will happen once it reaches the corresponding pruner node.

In order to estimate the cost of AQP, we need to know, for each node $s$, the number $\Phi(s)$ of nodes $s^{\prime}$ for which the pruners $\operatorname{pr}\left(s^{\prime}\right)$ appear in the subtree rooted at $s$ (i.e., $\operatorname{pr}\left(s^{\prime}\right) \in B(s)$ ). The basestation can derive $\Phi(s)$ (similarly to the derivation of $|B(s)|$ ). For random topologies, during the process 
of determining the prunees (see Section 4.1.2), nodes compute and forward this number to the base. The expected number of nodes in a subtree $B(s)$ rooted at $s$ not pruned by a pruner which is also in $B(s)$ is:

$$
K(s)=|B(s)|-\Phi(s) \cdot \frac{E_{1}+E_{2}}{E}
$$

The number $T_{\mathrm{AQP}}$ of transmitted packets during AQP can be estimated after replacing $|B(s)|$ by $K(s)$ in Eq. 1. Finally, the cost of AQP becomes:

$$
\operatorname{Cost}(\mathrm{AQP})=C_{T} T_{\mathrm{AQP}}+C_{R} R_{\mathrm{AQP}}+N\left(C_{S 1}+C_{S 2}\right)
$$

Distributed protocols Consider the distributed protocol DS1. In the first stage, nodes that qualify $P_{1}$ broadcast messages and nodes that qualify $P_{2}$ listen, potentially receive them, and formulate query results. Thus, $T_{\mathrm{DS} 1}^{1}=N \cdot \operatorname{Sel}\left(P_{1}\right)$ packets are sent. In addition, there is a listening cost for $L_{\mathrm{DS} 1}^{1}=N \cdot \operatorname{Sel}\left(P_{2}\right)$ packets and a reading cost for $R_{\mathrm{DS} 1}^{1}=\left(N \cdot \operatorname{Sel}\left(P_{2}\right)\right) \cdot\left(\rho \cdot \operatorname{Sel}\left(P_{1}\right)\right)$ packets. Thus the total cost of DS1 in the first stage (including sampling) is:

$$
\operatorname{Cost}^{1}(\mathrm{DS} 1)=C_{T} T_{\mathrm{DS} 1}^{1}+C_{L} L_{\mathrm{DS} 1}^{1}+C_{R} R_{\mathrm{DS} 1}^{1}+N\left(C_{S 1}+C_{S 2}\right)
$$

The corresponding cost of DS2 can be derived by swapping $P_{1}$ and $P_{2}$ in Eq. 5. Observe that the reading costs of DS1 and DS2 are identical. Since transmitting a message is much more expensive than listening ${ }^{6}$ (i.e., $C_{T}>C_{L}$ ), DS1 should be preferred to DS2 if $\operatorname{Sel}\left(P_{1}\right)<\operatorname{Sel}\left(P_{2}\right)$ (and vice-versa).

The second stage of all distributed protocols is similar to AQB; join results are sent up the routing tree and no filtering is performed. Therefore, to compute the packet transmissions $T_{\mathrm{DS} 1}^{2}$ during the second stage of DS1, it suffices to adjust Eq. 1, substituting $E$ by $E^{\prime}$; the expected query results generated by a node. $E^{\prime}$ equals $\operatorname{Sel}\left(P_{2}\right) \cdot\left(\rho \cdot \operatorname{Sel}\left(P_{1}\right)\right)$ (i.e., component of $\left.R_{\mathrm{DS} 1}^{1}\right)$. In addition, $C$ becomes $C^{\prime}$, since the capacity of packets now changes (join results are transmitted instead of

\footnotetext{
${ }^{6}$ We employ low-power idle listening [15], where a node listens only for a short time interval for potential messages. See Table 1 for some typical operation costs.
} 
single node locations, as in $\mathrm{AQB} / \mathrm{AQP})$. The reading cost $R_{\mathrm{DS} 1}^{2}$, during the second stage is derived by removing from $T_{\mathrm{DS} 1}^{2}$ the root's transmissions. Overall, the cost of DS1 is given below:

$$
\operatorname{Cost}(\mathrm{DS} 1)=\mathrm{Cost}^{1}(\mathrm{DS} 1)+C_{T} T_{\mathrm{DS} 1}^{2}+C_{R} R_{\mathrm{DS} 1}^{2}
$$

We conclude the analysis by considering the cost of the protocol DS1', which is described at the end of Section 4.1.3. This protocol asks nodes that qualify $P_{1}$ to transmit messages and all nodes in the network to listen and receive messages unconditionally. Only nodes that receive messages sense and verify $P_{2}$. In this case, $T_{\mathrm{DS} 1^{\prime}}^{1}=T_{\mathrm{DS} 1}^{1}$, but $L_{\mathrm{DS} 1^{\prime}}^{1}=N$ and $R_{\mathrm{DS} 1^{\prime}}^{1}=N \cdot\left(\rho \cdot \operatorname{Sel}\left(P_{1}\right)\right)$; i.e., the listening and reading costs increase. On the other hand, the sampling cost is reduced to $S A_{\mathrm{DS} 1^{\prime}}^{1}=N\left(C_{S 1}+\left(\rho \cdot \operatorname{Sel}\left(P_{1}\right)\right) C_{S 2}\right)$ and the overall cost of the first stage is:

$$
\operatorname{Cost}^{1}\left(\mathrm{DS}^{\prime}\right)=C_{T} T_{\mathrm{DS}^{\prime}}^{1}+C_{L} L_{\mathrm{DS}^{\prime}}^{1}+C_{R} R_{\mathrm{DS}^{\prime}}^{1}+S A_{\mathrm{DS}^{\prime}}^{1}
$$

By comparing the first-stage costs of DS1,DS2,DS1', and DS2' (i.e., the symmetric of DS1'), the optimizer can determine the most appropriate distributed protocol based on the selectivities of the predicates and the sampling costs.

\section{Extensibility}

In this section, we discuss extensions of spatial pattern queries and the issue of multiple query processing.

\subsection{Queries with temporal predicates}

The queries that we have seen so far apply to a particular time-snapshot of the network (i.e., a single epoch), looking for sensor combinations that qualify unary selections and binary spatial predicates. Analysts may also be interested in patterns that include temporal predicates between sensor readings. For example, consider the spatio-temporal join pattern query: "report cases, where a high temperature is sensed at most 5 seconds after a nearby low-humidity reading". In addition to the 
spatial constraint (nearby), qualifying pairs of readings should also satisfy a temporal constraint. Formally, Definition 1 of Section 3 can be enriched to include constraints $T_{i j}$ between pairs of variables $\left(v_{i}, v_{j}\right)$. The temporal constraint can be in the form of an interval (e.g., $\left.[0,5]\right)$ of the allowed time difference $v_{j} . t-v_{i} . t$, where $v . t$ denotes the time instant the sensor value that instantiates variable $v$ was sampled. Note that the spatial-only queries we have seen so far in fact hide a temporal constraint; in a qualifying combination of sensor readings, all readings should be taken within the same epoch.

The acquisitional and distributed protocols discussed in Section 4 can easily be extended for handling queries with temporal constraints. For AQB, we only need to maintain (at the basestation) a window of recent readings (defined by the longest time difference between query variables). In AQP, pruner nodes should keep track, for every prunee $s$ the last time a value that qualifies $P_{1}$ or $P_{2}$ was last seen in their neighborhood. Based on this information, a reading from $s$ can be pruned, if we know that there is no tuple that can potentially join with it. Finally, in the distributed protocols, the sensor nodes must keep in their memory a window of last sensed values qualifying the 'oldest' predicate (e.g., low humidity in the example query above). Readings that qualify the 'most recent' predicate (e.g., high temperature) are broadcast and joined with the buffers of their neighbors, where query results are computed and sent to the base.

\subsection{Monitoring the validity of query results}

There are cases, where query results remain valid for long time. For instance, once a hightemperature/low-humidity combination is being detected, it could stay valid for a long period. For such cases, continuously reporting the same result wastes resources. The only interesting information for long-lasting patterns is when they cease to be valid. Thus, an interesting problem is to monitor the validity of query results, while minimizing energy consumption. The distributed protocols are especially suitable for this purpose. As soon as a query result is identified by a node $s$, all other participant nodes are notified (by a simple broadcast from $s$ ). At the same time, $s$ becomes responsible of notifying the basestation for the invalidation of the pattern at some future time instant. While the values of the participant nodes satisfy the corresponding selections, the 
result remains valid. If a node $s_{i}$ violates its local selection $P_{i}$, it sends a message to $s$, which notifies the base.

\subsection{Multiple query optimization}

Multiple query optimization is an important issue for sensor networks, due to the high evaluation cost. For simplicity, assume that queries have a common routing tree. For extending protocol $\mathrm{AQB}$, we can apply the techniques of [1] that push down tables with all selection predicates that appear in the patterns. A simple adaptation of AQP is to compute and use at every node a set of prunees for each distance value which is a multiple of the radio range (i.e., one-hop prunees, two-hop prunees, etc.). Tuples that qualify selections are enriched with a bitmap indicating the set of queries for the selections of which they are valid. A pruner keeps track of the queries that apply in each prunee list and uses it to potentially filter tuples, relevant to these queries. The distributed protocols can be effective only when multiple queries share common predicates. On the other hand, (as suggested in [14]), in-network distributed evaluation of multiple queries may not be appropriate if their types and predicates vary greatly. A promising idea is to adopt a hybrid approach, where common selection predicates and low-selectivity joins are pushed in the network and expensive queries are left for evaluation at the basestation. In the future, we plan to study the optimization of multiple spatial pattern queries extensively.

\section{Experimental Evaluation}

In this section, we evaluate the efficiency of the proposed protocols on an experimental platform that simulates real sensor networks. Table 1 shows the components we consider when measuring query cost (taken from [15]). In all (but one) experiments, the selection predicates are applied on the cheapest to sense measurements, thus the sensing cost is negligible compared to communication/listening costs. We do not count the computational cost, since the operations involved in our protocols are cheap filters or distance checks. The packet size (excluding the header) was set to 30 bytes (typical for MICA motes [13]). Our protocols pack multiple events/messages in one packet, before transmitting them. The acquisitional protocols use 18-bit messages (node-id 
or coordinates plus 2 bits for indicating qualified predicates). The distributed protocols use 32-bit messages for sending pairs of node ids/coordinates to the root. As in REED [1], we assume longrunning queries and do not count the one-time cost of initializing the query in the sensor network. In each experiment, we run a protocol for 100 epochs and record the average cost per epoch.

\begin{tabular}{|l|r|}
\hline Operation & Cost (nAh) \\
\hline Transmitting a packet & 20 \\
Receiving a packet & 8 \\
Idle listening (for 1 ms) & 1.25 \\
Thermistor sample & 0.35 \\
Barometric pressure sample & 1.39 \\
Photoresistor sample & 3.43 \\
Infrared sample & 9.44 \\
$I^{2}$ CTemperature sample & 20.83 \\
\hline
\end{tabular}

Table 1: Costs of MICA operations

We experimented with the three network types described in Section 5, denoted by RANDOM, GRID, and LADDER. The default network size is $N=1024$ nodes. For all topologies, the root node of the routing/aggregation tree is chosen as the center of the network $[13,5]$. To generate the RANDOM network, we randomly placed nodes inside a square area of side $\sqrt{N}$ and set the radio range to 1.5 . These settings result in a network that is fully connected and not extremely dense, as shown in Figure 5. The average degree of a sensor node is 6.8, 4, and 3 in RANDOM, GRID, and LADDER topologies, respectively. The corresponding routing trees have heights 24,33 , and 258, respectively. Unless otherwise stated, the selectivities of unary predicates (i.e., $P_{1}, P_{2}$ ) are set to 0.05. For a single-hop binary join, these settings return 10-20 join results on average (depending on the network topology).

\subsection{Single-hop binary joins}

We first study the performance of the proposed protocols for low-selectivity single-hop binary join queries (with two selection predicates $P_{1}$ and $P_{2}$ ). Protocols AQB, AQP, and the distributed protocol (described in Section 4.1) are compared. By default, the selectivities of $P_{1}$ and $P_{2}$ are equal, so DS1 is equivalent to DS2; we simply denote either of them by DS. 


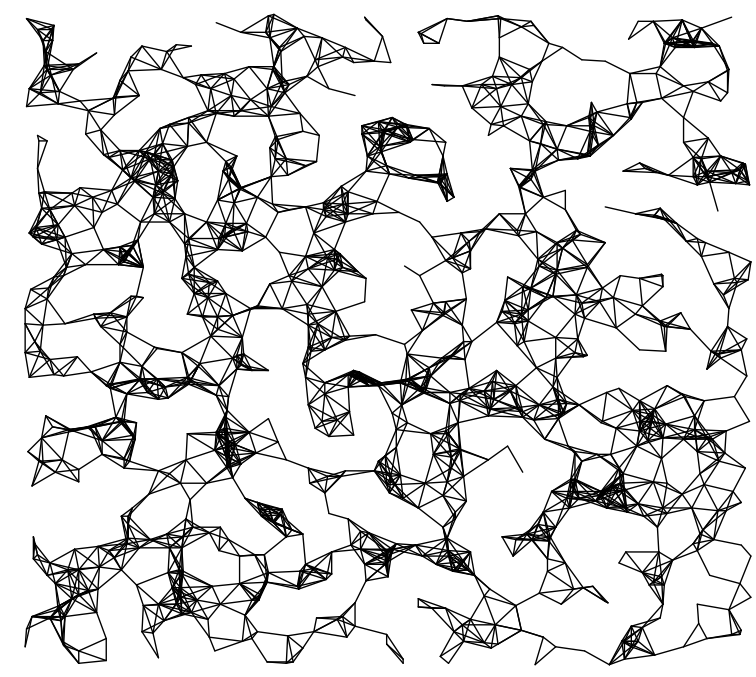

(a) network

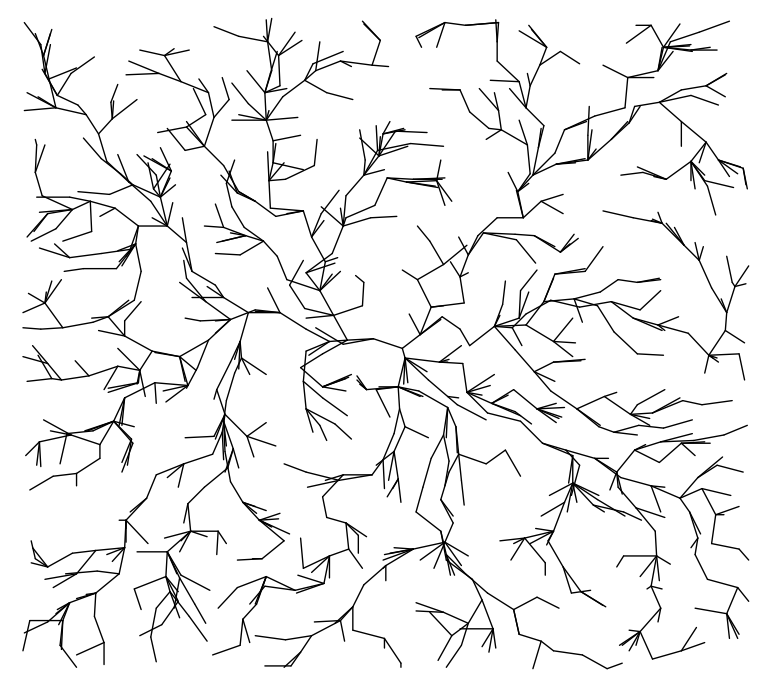

(b) routing tree

Figure 5: RANDOM topology

Figures 6a to $6 \mathrm{c}$ show the averaged costs (with error bars) of the three protocols as a function of the join selectivity. The join output size was controlled by tuning $\operatorname{Sel}\left(P_{1}\right)\left(=\operatorname{Sel}\left(P_{2}\right)\right)$. For joins with few results, Protocol DS is more efficient than AQB and AQP because pruner nodes (in AQP) are located several levels above their prunees and measurements that qualify the selections participate in very few or no join results. In GRID, the effectiveness of AQP is low because pruner nodes appear at high levels of the tree. On the other hand, in LADDER, pruning effectiveness is maximized, since each node has its pruner only 1-2 hops away. As the join output size increases, the energy consumption increases for all protocols, as more tuples are transferred to the base, but the relative performance of DS compared to AQB/AQP decreases, as the number of join results compared to the tuples that qualify either $P_{1}$ or $P_{2}$ increases. Eventually, DS becomes worse than the acquisitional protocols, since all readings that qualify the selections participate join result and the join output size well exceeds number of tuples that qualify either selection. Figure $6 \mathrm{~d}$ validates the accuracy of the cost models, presented in Section 5. We applied equations in Section 5 to estimate the costs of the protocols for each query $Q$ on the RANDOM network and averaged the error $\frac{|e s t(Q)-a c t(Q)|}{\operatorname{act}(Q)}$ for all queries having the same join selectivity $(\operatorname{est}(Q)$ and $\operatorname{act}(Q)$ are the estimated and actual costs, respectively). Observe that the error is quite low (less than 10\%) and decreases with the output size, since queries with more result have less randomness. We note that 
error for GRID and LADDER (not shown) is even smaller.

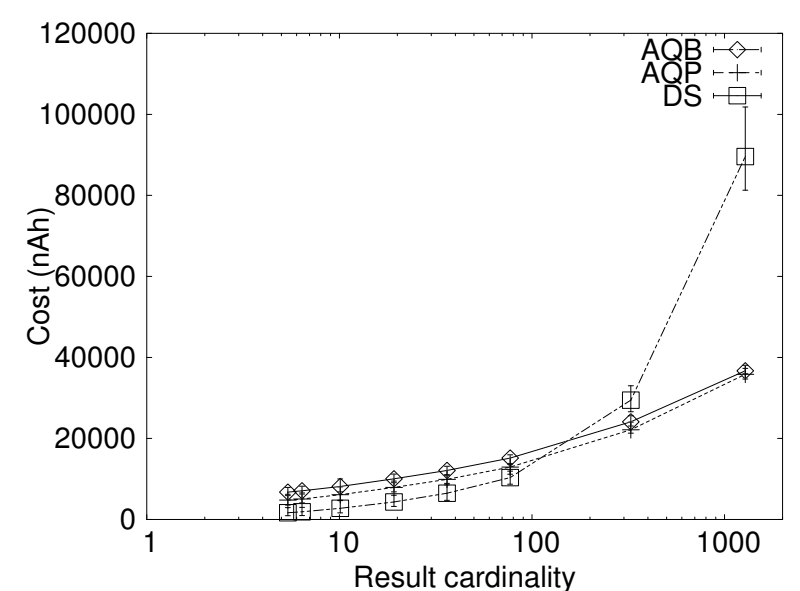

(a) RANDOM topology

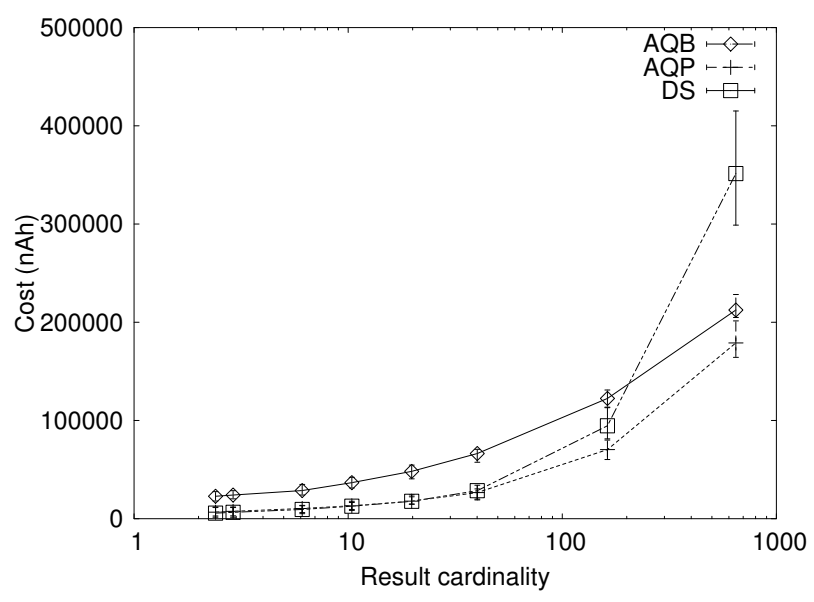

(c) LADDER topology

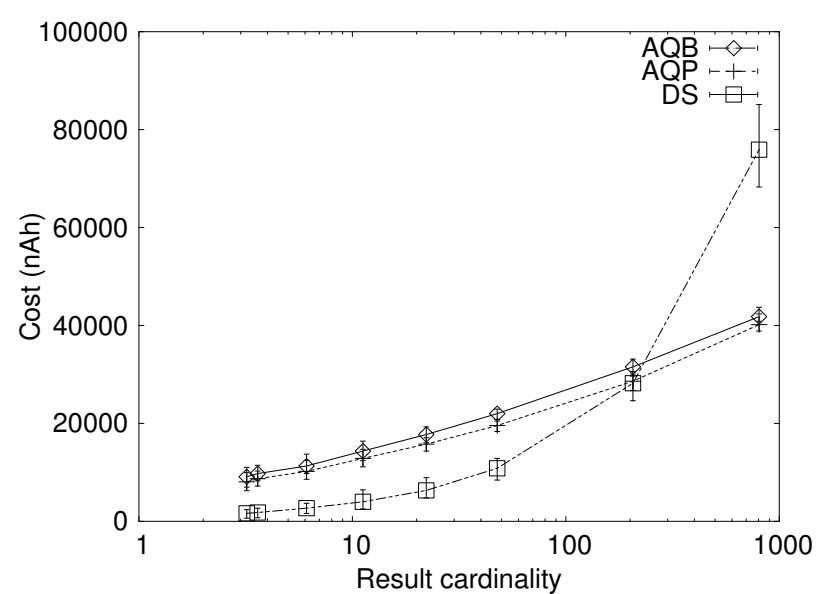

(b) GRID topology

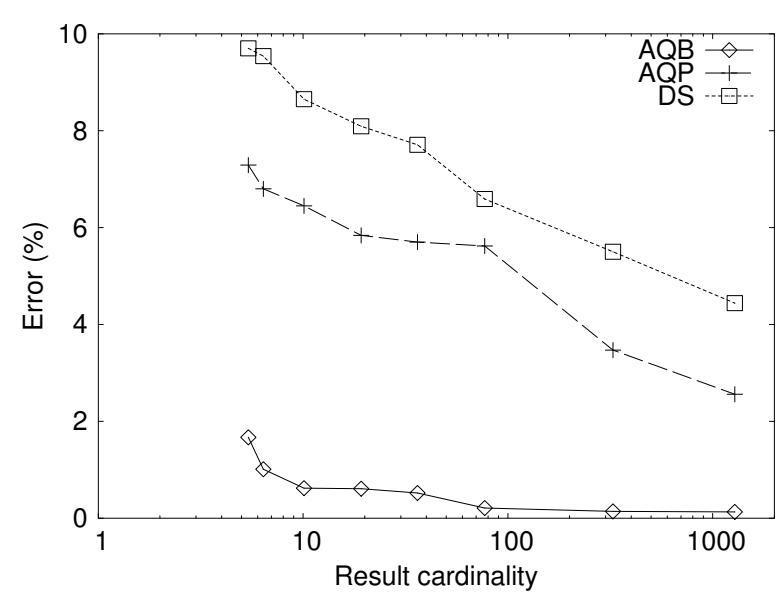

(d) estimation accuracy

Figure 6: Effect of join output size

Table 2 provides statistics about the effectiveness of pruner nodes in AQP and sheds some light to the unstable performance of the protocol at different network topologies. The table distributes the 1024 nodes of each network into classes based on the percentage of hops saved if their tuples are pruned by AQP. For instance, if a node $s$ falls into the $80 \%-100 \%$ class, then the quantity $\frac{\text { hops between } \operatorname{pr}(s) \text { and the base }}{\text { hops between } s \text { and the base }}$ (i.e., the path ratio saved if a tuple from $s$ was pruned by $\operatorname{pr}(s)$ ) is between 0.8 and 1. As explained, pruner nodes are close to their prunees in the LADDER network, resulting in large cost savings. On the other hand, in the GRID network, the chances that a message is not pruned until it travels a long way are high. Nodes in GRID are not clustered thus there is a high 
probability that a neighborhood is split into different subtrees. The effectiveness of pruners in RANDOM is in-between the two other topologies.

\begin{tabular}{|l|r|r|r|}
\hline Ratio(\%) & RANDOM & GRID & LADDER \\
\hline $80-100$ & 414 & 179 & 994 \\
$60-80$ & 250 & 280 & 16 \\
$40-60$ & 165 & 243 & 9 \\
$20-40$ & 105 & 190 & 5 \\
$0-20$ & 90 & 132 & 0 \\
\hline
\end{tabular}

Table 2: Number of nodes for each hops-saving class (protocol AQP)

Next, we verify the assertion that AQP and DS achieve better cost balancing than AQB among different nodes. Figure 7 shows the average cost per node as a function of node's level in the routing tree, in the RANDOM and GRID topologies (the plot for the LADDER network is similar). In general, sensor nodes at higher levels receive and forward more data so they have larger burden. DS and AQP have better balancing, since they manage to eliminate tuples that do not participate in join results early, either by computation of the exact join results (DS) or by filtering tuples at pruner nodes (AQP).

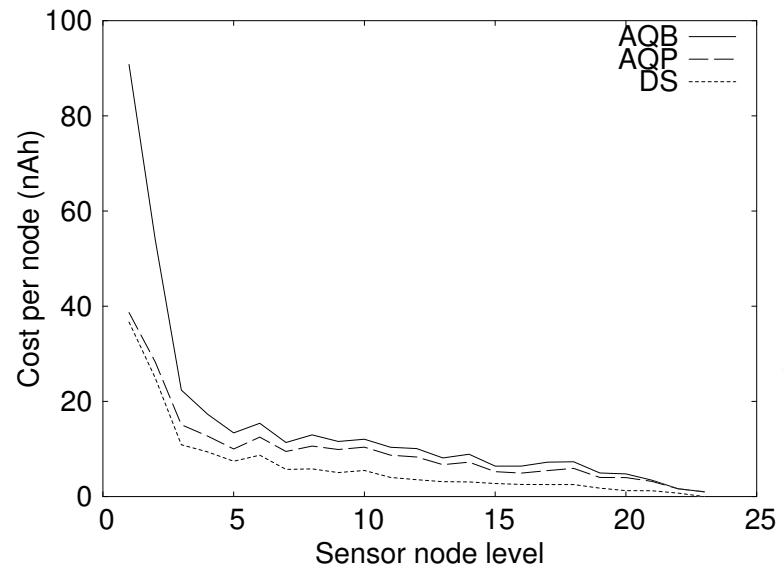

(a) RANDOM topology

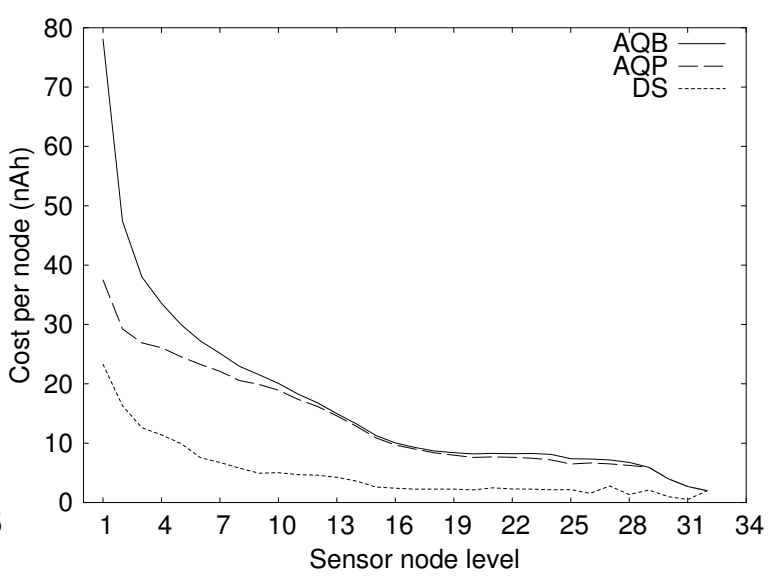

(b) GRID topology

Figure 7: Cost balancing of sensor nodes

We also tested the effect of the network density to the relative performance of the protocols. We gradually increased the communication range in the GRID and LADDER networks and measured the costs of the three protocols. Figure 8 plots the average energy consumption as a function of the average node degree in the two networks. In this experiment, we keep $P_{1}$ and $P_{2}$ constant and 
set $c$ to the increasing communication range, thus the join results increase with the density. As the network density increases, the height of the routing tree decreases and AQB becomes more efficient. The cost of AQP follows a similar trend in GRID networks, since the effectiveness of pruners is low. In LADDER networks, on the other hand, the cost of AQP increases initially with the density, as the effectiveness of pruners drops (due to the increase of neighborhood sizes and join results), and then drops (due to the high impact of the tree's height decrease) With the increase of density, protocol DS spends more energy in the distributed stage as more nodes receive messages from their neighbors. In addition, it generates and forwards more join results in the second stage. The additional costs are compensated by the tree height decrease, thus, the performance of DS

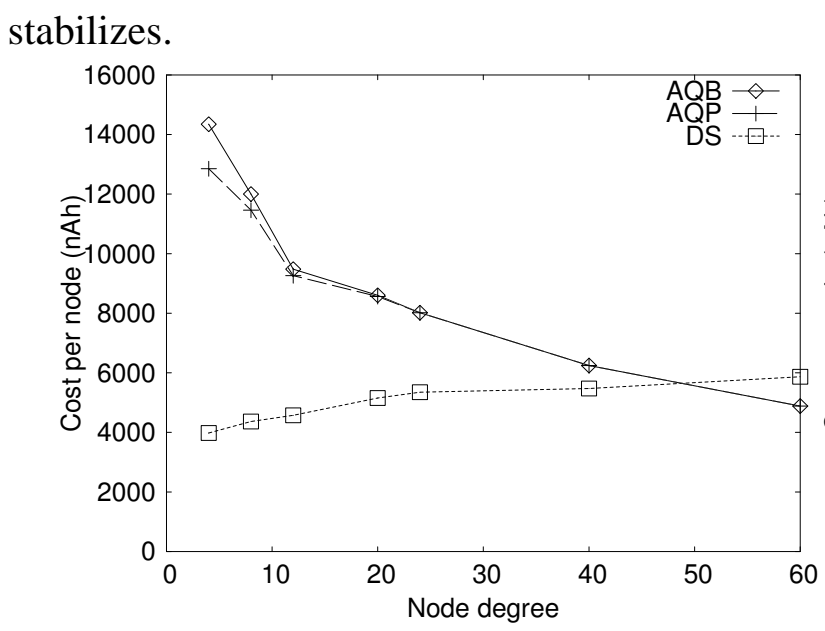

(a) GRID topology

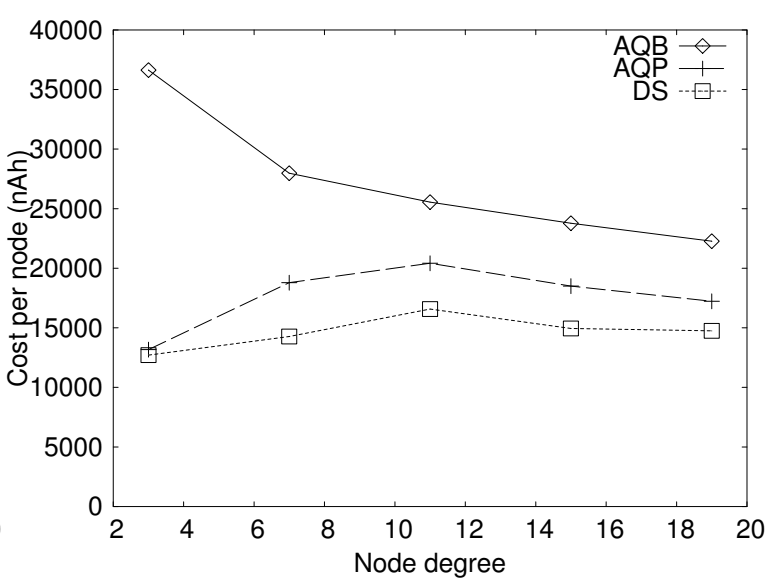

(b) LADDER topology

Figure 8: Effect of network density

In the next set of experiments, we test the performance of the protocols on the RANDOM topology. Figure 9a shows the cost of the protocols as a function of number of nodes, while keeping the network density fixed. Note that the cost difference between the protocols is not greatly affected by the network size. So far, we have assumed that $P_{1}$ and $P_{2}$ have the same selectivity. We now test the effect of unbalanced selectivities at the selection predicates (Figure 9b). For this experiment, we kept the product of the two selectivities fixed and varied the ratio $r=\operatorname{Sel}\left(P_{2}\right) / \operatorname{Sel}\left(P_{1}\right)$. For various values of $r$ we plot the energy consumption by the different protocols. Since $\operatorname{Sel}\left(P_{1}\right) \neq$ $\operatorname{Sel}\left(P_{2}\right)$, we split protocol DS to DS1 and DS2. DS1 is more efficient than DS2 for $r>1$ and 
its cost decreases with $r$. As $r$ increases, the number of sensors that qualify $P_{1}$ decreases, and so do the transmitted messages by DS1. Although the number of listeners (i.e., nodes that qualify $P_{2}$ ) increases, the listening (and reading) cost is significantly lower than the transmission cost (see Table 1), thus the overall cost of DS1 drops. On the other hand, the cost of DS2 (slightly) increases with $r$, due to the increased number of transmissions. Acquisitional protocols become more expensive with $r$, since the number of sensors that qualify either $P_{1}$ or $P_{2}$ increases. $^{7}$

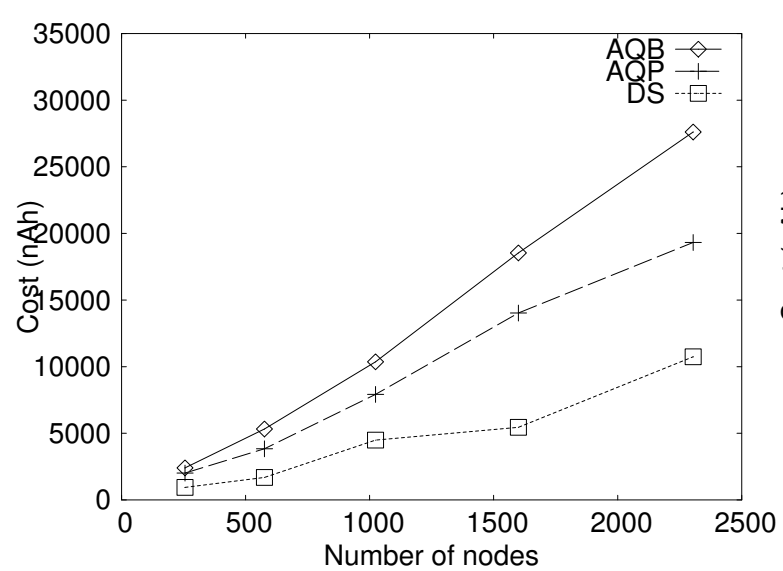

(a) scalability

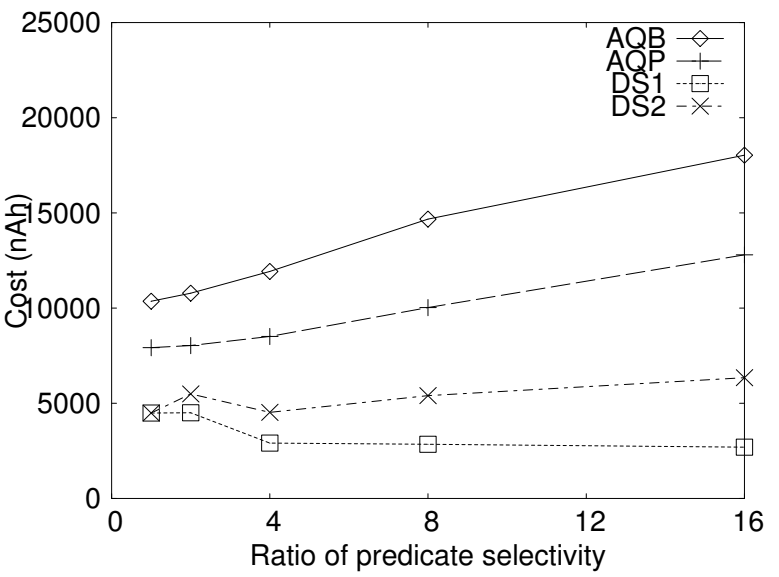

(b) effect of $\frac{\operatorname{Sel}\left(P_{2}\right)}{\operatorname{Sel}\left(P_{1}\right)}$

Figure 9: Net. size / predicate skew (RANDOM)

A natural question for advanced sensor network protocols is whether any additional operations performed by them affect the data loss rate, due to communication errors. We first evaluate the effect the packet loss rate has on the performance of the algorithms (Figure 10a). As the plot shows, the relative performance of the methods is not affected by this factor. Figure 10b shows the join output size as a function of packet loss rate. Observe that similar number of results are detected by different protocols. Thus, the functionality of the protocols does not affect the result loss rate in lossy networks. On the other hand, even with relatively low packet loss rates (10\%) a large percentage of results is not detected. This is expected, as the probability of a join result (or a component tuple in a join result) to reach the basestation decreases exponentially with the number of hops the message needs to travel. The reliability of the network can be increased if during the acquisition phase nodes request an acknowledgment from their parents when they sent data to

\footnotetext{
${ }^{7}$ For a fixed product $\operatorname{Sel}\left(P_{2}\right) \cdot \operatorname{Sel}\left(P_{1}\right)$, the probability for a sensor to qualify either $P_{1}$ or $P_{2}$ (i.e., $1-(1-$ $\left.\left.\operatorname{Sel}\left(P_{1}\right)\right)\left(1-\operatorname{Sel}\left(P_{2}\right)\right)\right)$ is minimized when $\operatorname{Sel}\left(P_{2}\right)=\operatorname{Sel}\left(P_{1}\right)$ and increases with $r$.
} 
them. Alternatively, multi-path routing techniques paired with duplicate elimination mechanisms (e.g., $[5,16])$ could be applied. Intuitively, protocol DS is more appropriate for multi-path routing than AQB (or AQP), since (i) the amount of transferred data is low as only (rare) join results are routed and (ii) the pruner nodes of $\mathrm{AQP}$ will be less effective, since tuples from prunees may find other paths to the root. A natural technique for improving network reliability is to introduce redundancy in message transmission. ${ }^{8}$

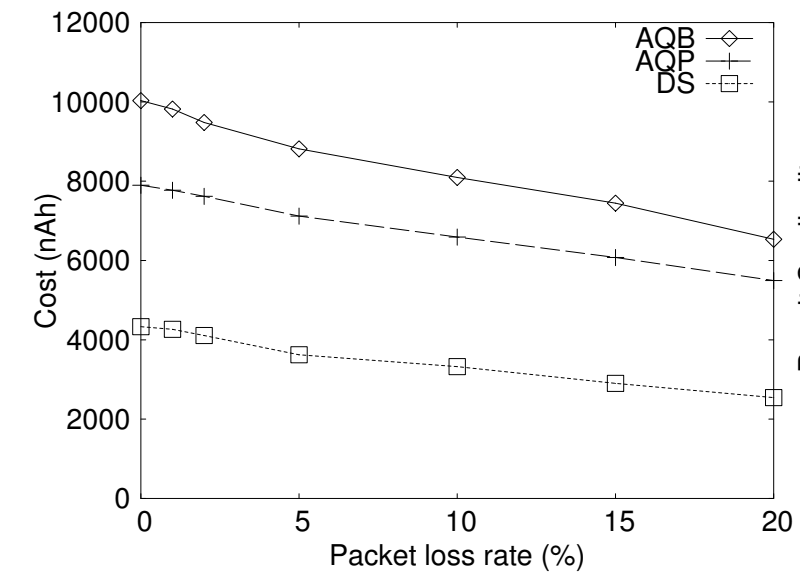

(a) cost

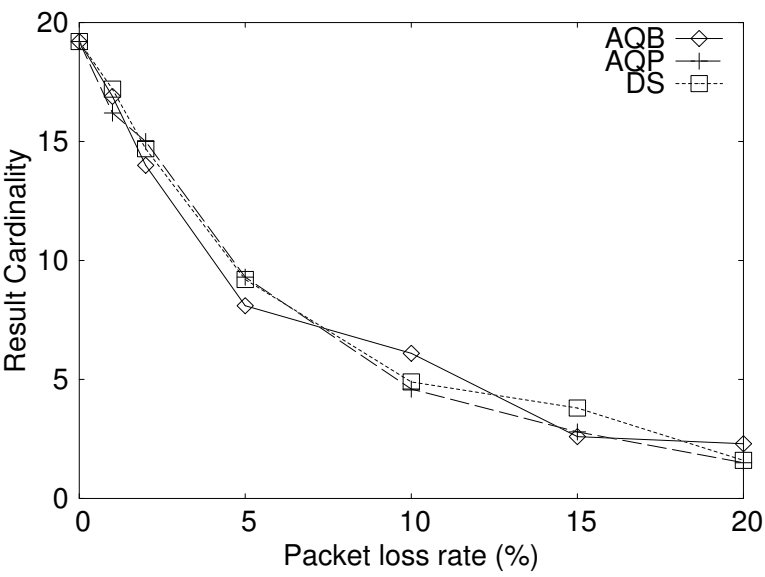

(b) result cardinality

Figure 10: Effect of packet loss rate (RANDOM)

We now examine a case where sampling data is significantly expensive. We consider a query, where $P_{1}$ applies on barometric pressure, $P_{2}$ applies on $I^{2} C$ temperatures and the selectivity of each predicate is 0.05 . We compare the original protocol DS, with the variant of it, described in Section 4.1, which asks all nodes to unconditionally listen to messages from nodes that qualify $P_{1}$. Only nodes that receive messages apply sampling to verify the selection condition of $P_{2}$. We denote this protocol by DS'. Table 3 displays the cost-breakdown of the join for DS and DS'. Observe that protocol DS ${ }^{\prime}$ has higher packet receiving cost and idle listening cost, but it has a much lower cost on sensing the expensive measurement. In total, protocol $\mathrm{DS}^{\prime}$ outperforms protocol DS. In general, $\mathrm{DS}^{\prime}$ should be preferred to DS when (i) sampling for either $P_{1}$ or $P_{2}$ is very expensive

\footnotetext{
${ }^{8}$ Let $p$ be the packet loss rate of each sensor node. Consider a fixed retransmission scheme, where each sensor node repeats $z$ times its transmitting/receiving/idle listening operation. Thus, the probability of successfully transmitting a packet (between neighbors) increases rapidly from $(1-p)$ to $\left(1-p^{z}\right)$. For example, suppose that the original packet loss rate is $p=0.2$ (i.e., successful transmission probability of 0.8 ). At $z=2(z=3)$, we double (triple) the energy consumption of sensors and the successful transmission probability (between neighbors) rises to 0.96 (0.992). Thus, we are able to achieve a very high successful transmission probability, with only a small factor $z$ in energy consumption.
} 
and should not be performed unconditionally or (ii) either $\operatorname{Sel}\left(P_{1}\right)$ or $\operatorname{Sel}\left(P_{2}\right)$ is close to $100 \%$; the majority of nodes qualify the predicate, so sensing should follow listening.

\begin{tabular}{|c|r|r|}
\hline \multirow{2}{*}{ Operation } & \multicolumn{2}{|c|}{ Average nodes / epoch } \\
\cline { 2 - 3 } & Protocol DS & Protocol DS \\
\hline \hline Transmitting a packet & 162.8 & 162.8 \\
\hline Receiving a packet & 126.6 & 461.9 \\
\hline Idle listening & 49.9 & 1024 \\
\hline Sensing barom. pressure & 1024 & 1024 \\
\hline Sensing $I^{2} C$ temp. & 1024 & 16.2 \\
\hline \hline Total cost (nAh) & 27084.5 & 9992.0 \\
\hline
\end{tabular}

Table 3: Cost breakdown for a query with expensive predicates (RANDOM)

\subsection{Complex joins}

In this section, we evaluate the effectiveness of the protocols described in Section 4.2 for spatial pattern queries with variables forming a star graph topology. Figure 11 shows the cost of the protocols as a function of number of border nodes, after fixing the selectivities of both predicates $P_{C}$ and $P_{B}$ to 0.1 . When the number of border nodes increases, only DSB and DSC achieve significant cost reduction. For queries with many border nodes, very few results are generated and the level-off costs of DSB and DSC indicate the cost of the distributed phase. DSB is slightly cheaper than DSC, because DSC requires more nodes to transmit packets in the distributed phase. The next experiment evaluates the protocols by varying the selectivities of $P_{C}$ and $P_{B}$. Figure 12a shows the cost of the protocols as a function of $P_{C}$ 's selectivity, with 3 border nodes and $\operatorname{Sel}\left(P_{B}\right)=0.05$. DSC has the best performance at very small values of $\operatorname{Sel}\left(P_{C}\right)$. DSB starts outperforming the other protocols as $\operatorname{Sel}\left(P_{C}\right)$ increases. Figure $12 \mathrm{~b}$ shows the cost of the protocols as a function of $\operatorname{Sel}\left(P_{B}\right)$, for queries with 3 border nodes and $\operatorname{Sel}\left(P_{C}\right)=0.05$. The situation is reversed in this case. DSB has the best performance at low values of $\operatorname{Sel}\left(P_{B}\right)$, while DSC becomes the best protocol as the number of border nodes increases. 


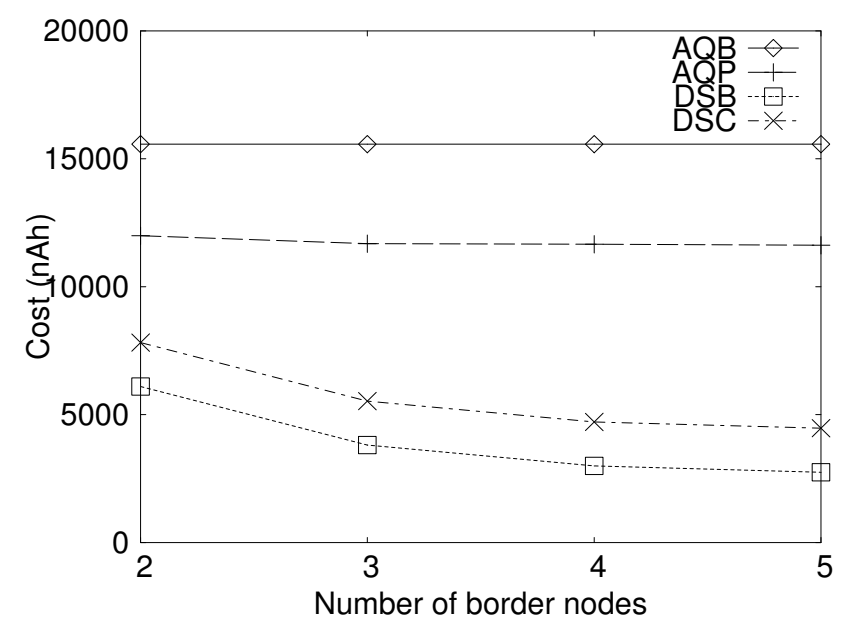

Figure 11: Cost as a function of number of border nodes, RANDOM topology

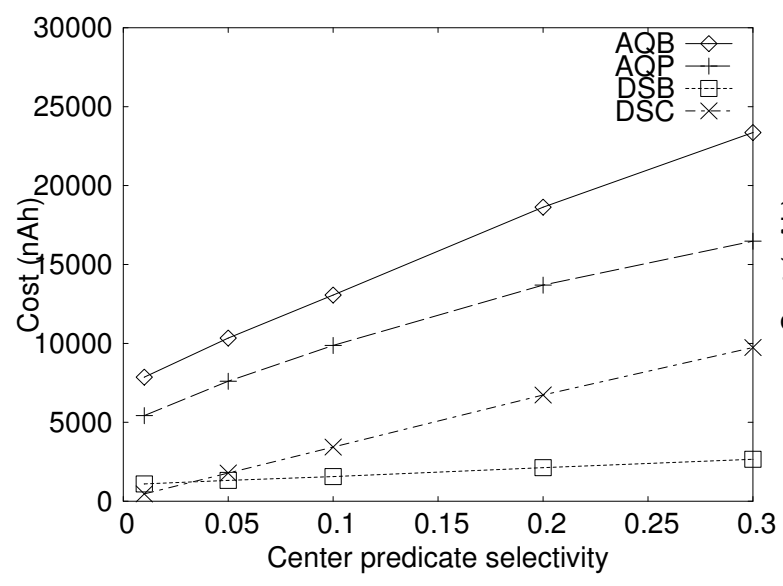

(a) varying $\operatorname{Sel}\left(P_{C}\right)$

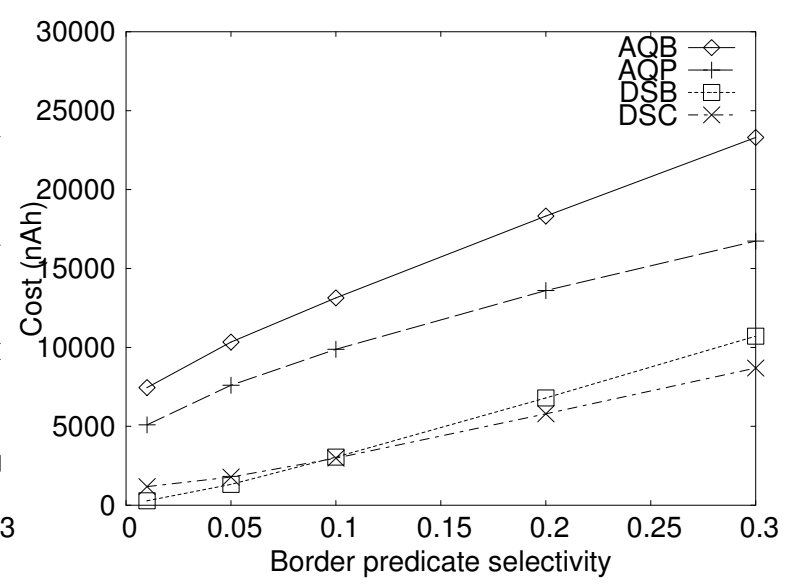

(b) varying $\operatorname{Sel}\left(P_{B}\right)$

Figure 12: Effect of selectivity (RANDOM)

\subsection{Multi-hop queries}

We now study the performance of the protocols for multi-hop binary join queries. In protocol $\mathrm{BD}, x$ is set to $\lambda / 2$. Figure 13 plots the costs as a function of join distance, on all three network topologies. In the RANDOM network, acquisitional protocols outperform distributed protocols for join distances greater than one hop. The result for GRID topology is similar except that acquisitional protocols start outperforming the distributed ones at a longer join distance. In the LADDER network, although the distributed protocols perform better than AQB, protocol AQP maintains the good performance it has at single-hop joins for multi-hop queries and greatly outperforms the 
distributed methods. The effectiveness of pruners remains high due to the linearity of the topology. Note that the bidirectional protocol (BD) does not have large performance difference than the purely distributed protocol. It turns out that BD has high packet reading cost, since intermediate nodes collect messages unconditionally. In addition, BD generates many duplicate join results which increase the cost of transmitting them to the basestation. In summary, acquisitional protocols are favorable for multi-hop queries, due to the extreme cost of flooding the selection results at long ranges.

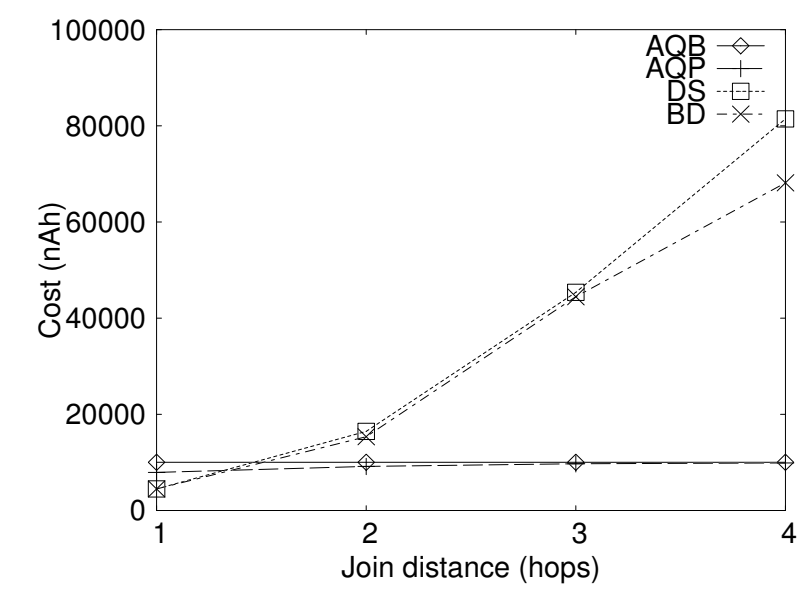

(a) RANDOM topology

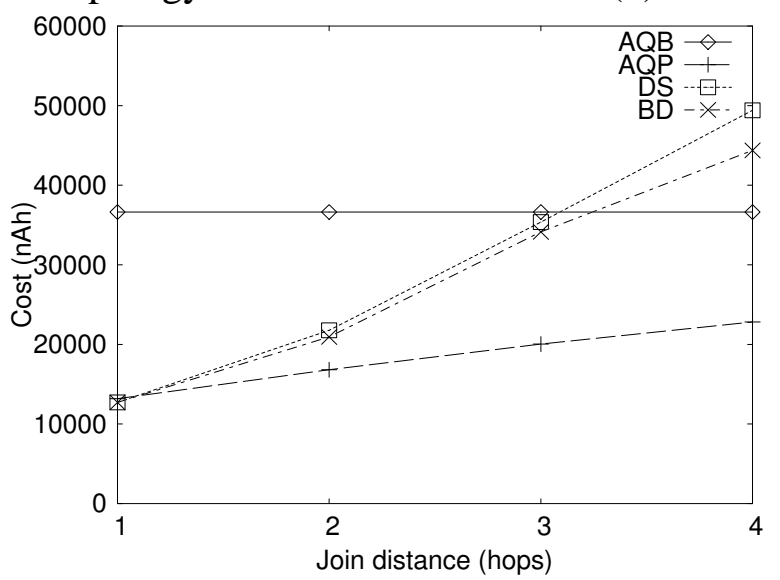

(c) LADDER topology

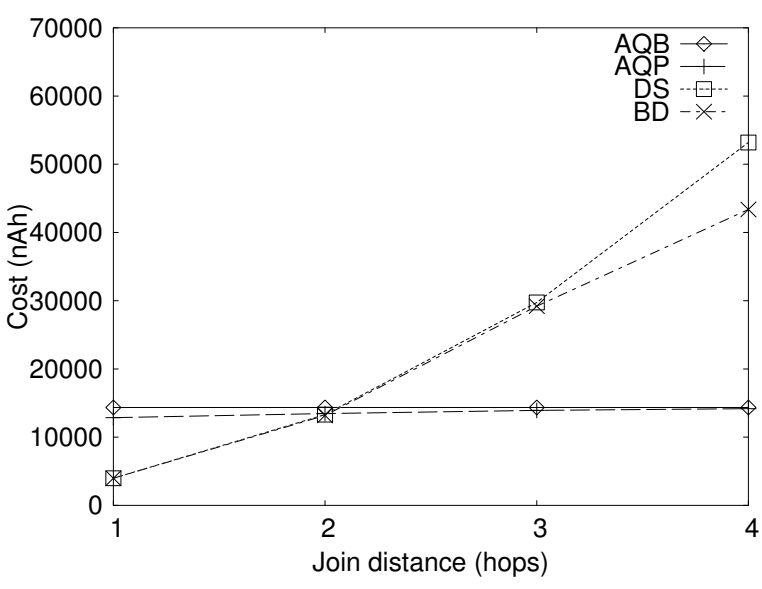

(b) GRID topology

Figure 13: Cost as a function of join distance

Finally, we verify the trade-off of disseminating continuous queries in a sensor network and applying in-network filtering or evaluation, as opposed to continuously and unconditionally acquiring measurements, and evaluating queries at the basestation. Table 4 shows the costs of the various 
protocols for disseminating queries, creating the routing tree, and determining non-trivial filters (i.e., prunee information by AQP). Observe that the base dissemination cost of the protocols (excluding prunee computation by $\mathrm{AQP}$ ) is relatively low and can be compensated if the query runs for a long enough period (e.g, $>10$ epochs), especially when $\operatorname{Sel}\left(P_{1}\right)$ and $\operatorname{Sel}\left(P_{2}\right)$ are small. On the other hand, the cost for computing the pruner/prunee information by AQP can be very high (especially for multi-hop queries). LADDER is the only type of network that especially favors $\mathrm{AQP}$, not only in terms of query performance, but also due to its low initialization cost. Although GRID has a smaller fanout (i.e. smaller neighbors), pruners have larger distance from prunees (when compared to RANDOM). Thus, the one-time dissemination cost of GRID is not low.

\begin{tabular}{|c|c|c|c|c|c|c|}
\hline \multirow{2}{*}{\multicolumn{3}{|c|}{ Base cost by AQB/AQP/DS }} & \multicolumn{4}{|c|}{ Extra cost by AQP } \\
\hline & & & \# hops & RANDOM & GRID & LADDER \\
\hline RANDOM & GRID & LADDER & 1 & $181 \mu \mathrm{Ah}$ & $165 \mu \mathrm{Ah}$ & $27 \mu \mathrm{Ah}$ \\
\hline $76 \mu \mathrm{Ah}$ & $53 \mu \mathrm{Ah}$ & $45 \mu \mathrm{Ah}$ & 2 & $673 \mu \mathrm{Ah}$ & $612 \mu \mathrm{Ah}$ & $116 \mu \mathrm{Ah}$ \\
\hline & & & 4 & $2767 \mu \mathrm{Ah}$ & $2690 \mu \mathrm{Ah}$ & $503 \mu \mathrm{Ah}$ \\
\hline
\end{tabular}

Table 4: Cost for query dissemination

\section{Conclusions}

In this paper, we studied the evaluation of spatial pattern queries, which output combinations of sensor readings qualifying unary selection predicates and pairwise distance constraints. We proposed protocols that can achieve significant performance savings compared to a simple acquisitional approach that performs filtering based only on the unary selections. An improved acquisitional protocol places join filters in the routing tree that eliminate sensor readings that do not qualify the distance constraints. A distributed protocol (with variants for multi-way or multi-hop queries) performs in-network computation of the results, before sending them to the user. We presented cost models that accurately estimate the costs of all evaluation protocols. Experimental studies suggest that the distributed techniques perform best for low-selectivity queries with single-hop distance predicates, whereas acquisitional protocols should be preferred for multi-hop or high-selectivity queries. In the future, we plan to study alternative spatial pattern queries that capture advanced characteristics such as the shape and distribution of sensor values. Regarding 
continuous query evaluation, we will continue to explore the approach in Section 6.2 for reducing energy consumption by saving notifications of identical spatial patterns in consecutive epochs.

\section{References}

[1] D. J. Abadi, S. Madden, and W. Lindner. REED: Robust, Efficient Filtering and Event Detection in Sensor Networks. In Proc. of VLDB, 2005.

[2] B. J. Bonfils and P. Bonnet. Adaptive and Decentralized Operator Placement for In-Network Query Processing. In Proc. of IPSN, 2003.

[3] P. Bonnet, J. Gehrke, and P. Seshadri. Towards Sensor Database Systems. In Proc. of MDM, 2001.

[4] D. Chu, A. Deshpande, J. Hellerstein, and W. Hong. Approximate Data Collection in Sensor Networks using Probabilistic Models. In Proc. of ICDE, 2006.

[5] J. Considine, F. Li, G. Kollios, and J. W. Byers. Approximate Aggregation Techniques for Sensor Databases. In Proc. of ICDE, 2004.

[6] A. Deligiannakis, Y. Kotidis, and N. Roussopoulos. Compressing Historical Information in Sensor Networks. In Proc. of ACM SIGMOD, 2004.

[7] A. Deligiannakis, Y. Kotidis, and N. Roussopoulos. Hierarchical In-Network Data Aggregation with Quality Guarantees. In Proc. of EDBT, 2004.

[8] A. Deshpande, C. Guestrin, S. Madden, J. M. Hellerstein, and W. Hong. Model-Driven Data Acquisition in Sensor Networks. In Proc. of VLDB, 2004.

[9] M. Hadjieleftheriou, N. Mamoulis, and Y. Tao. Continuous Constraint Query Evaluation for Spatiotemporal Streams. In Proc. of SSTD, 2007.

[10] C. Intanagonwiwat, R. Govindan, and D. Estrin. Directed Diffusion: A Scalable and Robust Communication Paradigm for Sensor Networks. In Proc. of MOBICOM, 2000. 
[11] Y. Kotidis. Snapshot Queries: Towards Data-Centric Sensor Networks. In Proc. of ICDE, 2005 .

[12] Y. Kotidis. Processing Proximity Queries in Sensor Networks. In International Workshop on Data Management for Sensor Networks, 2006.

[13] S. Madden, M. J. Franklin, J. M. Hellerstein, and W. Hong. TAG: A Tiny AGgregation Service for Ad-Hoc Sensor Networks. In Proc. of OSDI, 2002.

[14] S. Madden, M. J. Franklin, J. M. Hellerstein, and W. Hong. TinyDB: An Acquisitional Query Processing System for Sensor Networks. ACM TODS, 30(1):122-173, 2005.

[15] A. Mainwaring, D. Culler, J. Polastre, R. Szewczyk, and J. Anderson. Wireless Sensor Networks for Habitat Monitoring. In Proc. of WSNA, 2002.

[16] A. Manjhi, S. Nath, and P. B. Gibbons. Tributaries and Deltas: Efficient and Robust Aggregation in Sensor Network Streams. In Proc. of ACM SIGMOD, 2005.

[17] J. Paek, K. Chintalapudi, J. Cafferey, R. Govindan, and S. Masri. A Wireless Sensor Network for Structural Health Monitoring: Performance and Experience. In Proc. of the 2nd IEEE Workshop on Embedded Networked Sensors, 2005.

[18] A. Pandit and H. Gupta. Communication-Efficient Implementation of Range-Joins in Sensor Networks. In Proc. of DASFAA, 2006.

[19] M. A. Sharaf, J. Beaver, A. Labrinidis, and P. K. Chrysanthis. Balancing Energy Efficiency and Quality of Aggregate Data in Sensor Networks. VLDB J., 13(4):384-403, 2004.

[20] G. Simon, M. Maróti, Á. Lédeczi, G. Balogh, B. Kusy, A. Nádas, G. Pap, J. Sallai, and K. Frampton. Sensor network-based countersniper system. In Proc. of SenSys, 2004.

[21] A. Soheili, V. Kalogeraki, and D. Gunopulos. Spatial Queries in Sensor Networks. In Proc. of ACM GIS, 2005. 
[22] U. Srivastava, K. Munagala, and J. Widom. Operator Placement for In-Network Stream Query Processing. In Proc. of ACM PODS, 2005.

[23] Xiaoyan Yang and Hock-Beng Lim and M. Tamer Özsu and Kian-Lee Tan. In-network Execution of Monitoring Queries in Sensor Networks. In Proc. of ACM SIGMOD, 2007.

[24] Y. Yao and J. Gehrke. The Cougar Approach to In-network Query Processing in Sensor Networks. SIGMOD Record, 31(3):9-18, 2002.

[25] M. L. Yiu, N. Mamoulis, and S. Bakiras. Retrieval of Spatial Join Pattern Instances from Sensor Networks. In Proc. of SSDBM, 2007.

[26] H. Yu, E.-P. Lim, and J. Zhang. On In-network Synopsis Join Processing for Sensor Networks. In MDM, 2006.

[27] F. Zhao and L. Guibas. Wireless Sensor Networks: An Information Processing Approach. Elsevier/Morgan Kaufmann, 2004. 